\title{
Touristifizierung von Religion und Spiritualisierung von Tourismus. Erkundungen am Beispiel von religiösen Wanderwegen im Schwarzwald
}

\author{
Kornelia Sammet $(\mathbb{D} \cdot$ Uta Karstein
}

Eingegangen: 3. April 2021 / Überarbeitet: 28. August 2021 / Angenommen: 3. September 2021 / Online publiziert: 30. September 2021

(C) Der/die Autor(en) 2021

Zusammenfassung In den letzten Jahren hat religiös konnotiertes Reisen über den kirchlichen Kontext hinaus an Bedeutung gewonnen. Dies findet seinen Ausdruck auch in touristischen Angeboten und Marketingstrategien. Der Beitrag bearbeitet anhand des Wanderns als einer spezifischen Form der touristischen Bewegung in landschaftlich reizvoller Umgebung die Frage, wie durch den Tourismus auf Religiöses in Form von Artefakten, Orten und Wegen Bezug genommen und Religion touristisch recodiert wird, wodurch religiös gefärbte touristische Räume erzeugt werden.

Der Beitrag verfolgt eine differenzierungstheoretischen Perspektive, nach der Tourismus als ein sozialer Zusammenhang verstanden wird, der an vielen gesellschaftlichen Teilbereichen partizipiert und sie in kommunikativer Hinsicht mit Rückgriff auf den Code „,erlebnisförmige Erholung“ einer Recodierung unterzieht. Anhand von touristischem Material, das Wanderwege im Schwarzwald mit religiösen Wegmarken bewirbt, werden mit sequenzanalytischen Verfahren zwei typische kommunikative Muster von touristischen Bezugnahmen auf Religiöses rekonstruiert, nämlich die Musealisierung und die Kommodifizierung des Religiösen. In umgekehrter Perspektive werden religiöse Recodierungen des Touristischen analysiert, die als Kommunikation über Religion zu ihrer Kulturalisierung beitragen oder mit religiöser Kommunikation eine (Re-)Spiritualisierung von Landschaft betreiben. Abschließend werden die Fallanalysen zusammengeführt und ihre Anschlussfähigkeit an aktuelle religionssoziologische Konzepte diskutiert.

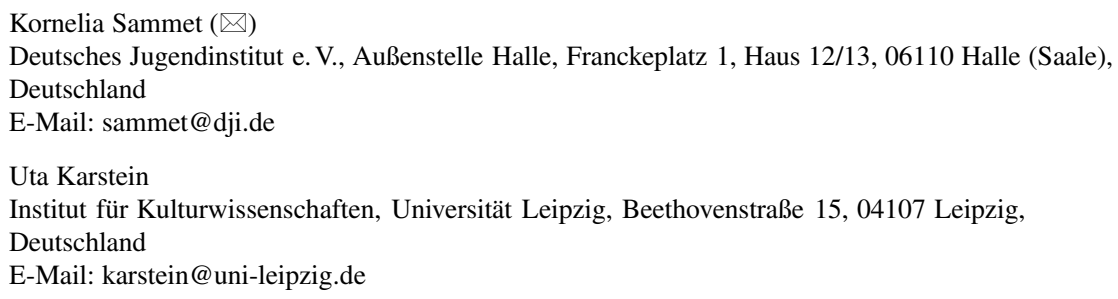


Schlüsselwörter Tourismus $\cdot$ Erlebnisförmige Erholung $\cdot$ Religiöse

Kommunikation · Popularisierung · Spiritualisierung · Raum · Kulturalisierung ·

Musealisierung · Kommodifizierung von Religion · Wandern · Recodierung ·

Schwarzwald

\title{
Touristification of religion and spiritualisation of tourism: sociological analyses of religious hiking trails in the Black Forest
}

\begin{abstract}
In the last years, religious travelling has gained importance beyond religious contexts. As a result, touristic actors have developed touristic services and marketing strategies with religious references. The paper deals with hiking as a specific form of touristic mobility in scenic surroundings. We argue that touristic communication produces touristic spaces and places by recoding sacred artefacts, buildings, sites and ways. Touristic communication defines what is worth seeing, presents touristic services and informs about ways to and in the touristic space

Referring to differentiation theories, the paper explores in a sociological perspective the touristification of sacred places and religious artefacts. Tourism participates in different social realms of society by recoding them with touristic semantics like "place of interest" and "sight-seeing". Based on data collected in the Black Forest in Germany, the paper analyses the description and presentation of walking and hiking trails. We reconstruct musealisation and commodification as two typical communicative patterns of touristification of religion. Additionally, we observe the religious recoding of touristic communication as culturalisation and as spiritualisation of touristic sites.
\end{abstract}

Keywords Tourism · Religious communication · Touristfication · Culturalisation of religion - Commodification of religion · Black Forest · Touristic

communication $\cdot$ Spiritualisation $\cdot$ Space $\cdot$ Recoding of religion $\cdot$ Hiking trails

\section{Einleitung: Einordnung und Fragestellung}

Der vorliegende Artikel widmet sich dem Verhältnis von Religion und Tourismus und damit einem Gegenstand, der bislang erst wenig in den Fokus der religionssoziologischen Forschung gerückt ist. Das Verreisen - ob individuell, mit der Familie oder als Teil einer Reisegruppe - ist zu einem Massenphänomen geworden. ${ }^{1}$ Davon zeugt auch die zunehmende Ausdifferenzierung, die dafür sorgt, dass es Angebote für jeden Geschmack und Geldbeutel gibt. In den letzten Jahren haben dabei auch religiös konnotierte Reiseformen über kirchliche Kreise hinaus zunehmende Aufmerksamkeit erfahren. Zu diesem Boom haben sicherlich Reiseberichte wie der des Autors und Comedians Hape Kerkelings beigetragen, der 2001 auf dem Jakobsweg unterwegs war und diesem damit zu unverhoffter Popularität verhalf (Kerkeling 2006). Zusammen mit den nicht nur im christlichen Kontext bekannten Wallfahrten

\footnotetext{
1 Allein in Deutschland verzeichnete die Tourismusbranche 2019 einen Umsatz von 35,4 Mrd. EUR (Graefe 2021).
} 
stellt das Pilgern traditionell eine der bedeutsamsten Formen religiös motivierten Reisens dar (Stausberg 2010). Diese Formen werden wir uns im Folgenden jedoch nicht anschauen. Im Zentrum unseres Interesses steht vielmehr das Wandern als eine typische touristische Bewegung im Raum und die semantischen Bezugnahmen auf diese Aktivität, durch die der bewanderte Raum in spezifischer Weise als touristischer Raum beschrieben und entworfen wird. Dem Verhältnis von Tourismus und Religion nähern wir uns zunächst von der Seite des Tourismus. In seinem Kontext avancierte das Wandern als eine Form der Bewegung, die zu Fuß in landschaftlich reizvoller Umgebung erfolgt, schon früh zu einer etablierten Praxis.

Am Beispiel des Wanderns wollen wir der Frage nachgehen, wie der Tourismus auf Religiöses Bezug nimmt, wie also Religion (z. B. in Gestalt religiöser Artefakte, Architekturen oder Religionsgeschichte) touristisch aufgegriffen und als Gegenstand touristischer Aktivitäten beschrieben wird. Diese Frage soll im Weiteren auch in umgekehrter Richtung verfolgt werden: Gibt es demnach religiöse Bezüge auf Touristisches, die Touristisches in Religiöses überführen?

Wir nähern uns dem Thema im Folgenden aus einer differenzierungstheoretischen Perspektive. Da sich Differenzierung nicht zuletzt kommunikativ manifestiert, fokussieren wir touristische und religiöse Kommunikation (Kap. 2). Nach der Darlegung der methodischen Vorgehensweise (Kap. 3) rekonstruieren wir verschiedene Formen der Touristifizierung von Religion auf der Basis von Datenmaterial zu Wanderwegen (Kap. 4). Diese Formen unterscheiden sich unter anderem dadurch, dass sie entweder Kommunikation über Religion darstellen oder selbst religiöse Kommunikation sind. Im abschließenden Kapitel führen wir die Fallanalysen zusammen und diskutieren ihre Anschlussfähigkeit an aktuelle religionssoziologische Konzepte.

\section{Theoretische Konzepte: Popularisierung, Kommunikation, Raum}

\subsection{Popularisierung und Differenzierung}

Unsere Analysen nehmen das Verhältnis von Religion und Tourismus in ihren wechselseitigen Bezügen in den Blick und verfolgen insofern eine differenzierungstheoretische Perspektive. Die moderne Gesellschaft ist demnach gekennzeichnet durch ihre sachliche (horizontale) Differenzierung und einen damit eng verknüpften neuen Inklusionsmodus. ${ }^{2}$ Während in der europäischen Vormoderne soziale Akteure vor allem über ihre Zugehörigkeit zu einem sozialen Stand in die Gesellschaft inkludiert wurden - wobei diese Inklusion in der Regel ihre ganze Person umfasste -, werden sie in der Moderne vor allem über ihre Publikumsrollen ${ }^{3}$ inkludiert, die sie in den jeweiligen gesellschaftlichen Teilbereichen einnehmen (können) (Stichweh 1988; Gerhardts 2001). Man ist Wählende im politischen System, Klient im Rechtssystem, Gläubige im Religionssystem, Zuschauer bzw. Besucherin im Kunstsystem etc. Da-

\footnotetext{
2 Der Aufsatz bezieht sich auf system- wie auch auf feldtheoretische Differenzierungskonzepte und spart eine grundlagentheoretische Diskussion zugunsten eines vorrangig pragmatischen Zugriffs aus.

3 „Publikum“ wird hier als eine grundlegende soziologische Kategorie verstanden und ist damit keineswegs beschränkt auf das Kunstfeld (Karstein und Zahner 2015).
} 
bei setzt man setzt sich in Komplementärrollen ins Verhältnis zu den Funktions- bzw. Leistungsrollen der jeweiligen Expert:innen der gesellschaftlichen Teilbereiche, wie den Ärzt:innen, Jurist:innen, Politiker:innen usw. (Stichweh 1994, S. 369 ff). Diese rollenförmige Inklusion ist immer nur partiell und spezifisch, aber vielfältig und damit ein wesentliches Moment moderner Individualitätserfahrung, insofern sich das Individuum als Kreuzung einer solchen Vielzahl sozialer Kreise konstituiert (Simmel 1992, S. 456-511). Und ihr kommt ein demokratischer Charakter zu, da diese Rollen prinzipiell Jedermann einnehmen kann. Die Rollen sind nicht exklusiv, d. h. von Standesmerkmalen oder ähnlichem abhängig, sondern offen oder zumindest von den formalen Voraussetzungen her niedrigschwellig. Dabei unterscheiden sich die Teilbereiche noch einmal hinsichtlich des konkreten Inklusionsmodus. Während beispielsweise das Recht oder die Medizin ein persönliches Klienten-ProfessionellenVerhältnis etablieren (vgl. Stichweh 1994, S. 368-375), ist für die Wirtschaft oder die Politik ein eher unpersönliches exit/voice-Modell typisch, in dem man zwischen verschiedenen Angeboten wählen kann, wobei die einzelnen Entscheidungen nur in aggregierter Form relevant werden, beispielsweise über signifikante Wählerwanderungen (Stichweh 1988, S. 270 ff.). Für den ästhetischen Bereich stellt sich das Ganze noch einmal komplexer dar, da das Kunstsystem mit unterschiedlichen Logiken operiert. Während sich am Pol der erweiterten Produktion tendenziell eher die exit/voice-Form finden lässt, findet sich am Pol der limitierten Produktion eher eine Logik, die sich derjenigen der Wissenschaft annähert, die auf den Zuspruch bzw. die Bestätigung durch Publikum nicht angewiesen ist bzw. es ablehnt, sich darüber zu legitimieren (Bourdieu 1999).

Popularisierung kann aus differenzierungstheoretischer Perspektive verstanden werden als ein Effekt, der sich einstellt, wenn breite Publikumsschichten an dem bereichsspezifischen (Experten-)Wissen und an entsprechenden Kompetenzen und Praktiken nicht nur partizipieren, sondern sie sich auch aktiv aneignen und in ihren eigenen Alltag integrieren. ${ }^{4}$ Beobachtbar ist dies unter anderem am Wissen über den menschlichen Körper, über mögliche Erkrankungen und deren Verhinderung mittels bestimmter (Hygiene-)Praktiken (z. B. Händewaschen). Dies wird befördert durch eine Aufbereitung des Wissens in einer Weise, die auf seine möglichst große Verbreitung zielt. Populärwissenschaftliche Bücher und Magazine sind hier ein typisches Beispiel, aber auch Druckerzeugnisse mit religiösen oder politischen Motiven, die die Präsenz dementsprechender Vorstellungen und affektiver Bindungen in der Bevölkerung verbreitern und stabilisieren sollten (Palatschek 2008; Kretschmann 2003). Spricht man von Populärkultur, hat man dementsprechend vor allem diejenigen Aktivitäten und Angebote des Kunstfeldes im Blick, die für ein möglichst breites Publikum produziert wurden. Typische Beispiele wären Musicals und Blockbuster. Allerdings können auch solche Werke einen Popularisierungsprozess durchlaufen, die zum Zeitpunkt ihrer Entstehung eher im avantgardistischen, exklusiven Bereich angesiedelt waren und ein vergleichsweise kleines Publikum hatten (Bourdieu 1999, S. 227-282). Aufgrund zunehmender Gewöhnung und Akzeptanz

\footnotetext{
${ }^{4}$ Dementsprechend verstehen wir - anders als Knoblauch (2009) - die beschriebenen Phänomene von Popularisierung nicht als Entgrenzung, da der jeweilige Bezugsrahmen bzw. Code erhalten bleibt. Auch durch Prozesse der Popularisierung handelt es sich weiterhin um Kunst, Gesundheit oder Politik usw.
} 
im Zeitverlauf setzt zuweilen eine zweite (und dritte) Rezeptionswelle ein, die den Bekanntheitsgrad erheblich steigert. Dies stellt die Grundlage einer weitergehenden Verwertung dar, für die als typische Beispiele etwa Motive bekannter Kunstwerke auf Radiergummis und Kaffeetassen, die dann im Museumsshop käuflich erworben werden können, oder ihre Verbreitung in Form günstiger Drucke als Wohnzimmerdekoration genannt werden können.

Tourismus ist in differenzierungstheoretischer Hinsicht nicht leicht zu bestimmen. Wir folgen in diesem Aufsatz dem Vorschlag von Andreas Pott, der Tourismus vor dem Hintergrund der Luhmannschen Systemtheorie nicht als ein eigenes soziales System konzeptualisiert, sondern als einen sozialen Zusammenhang, der an vielen anderen Teilbereichen partizipiert und sie in kommunikativer Hinsicht einer Recodierung unterzieht (Pott 2011). ${ }^{5} \mathrm{Zu}$ den für den Tourismus besonders relevanten Feldern zählen Sport, Kunst, Medizin oder Bildung. So gibt es unter anderem Aktivreisen (Klettertouren, Wanderungen, Segelturns), Bildungsreisen (Besichtigung regionaler oder kulturgeschichtlicher Besonderheiten) oder Gesundheitsreisen (Kuren, Wellness, Retreats). Wie weiter unten im Abschnitt 2.2 noch detailliert ausgeführt wird, wird dabei mit dem Code erlebnisförmige Erholung operiert. Bei einer Aktivreise etwa ginge es gerade nicht um Formen von Hochleistungs- oder Breitensport, sondern um körperliche Betätigungen, die aufgrund des damit verbundenen Ortswechsels und des nicht-alltäglichen Charakters einen besonderen Erlebniswert haben und - gerade deswegen - als erholsam gelten. Als touristische Recodierungen verstehen wir dementsprechend kommunikative Prozesse, durch die Gegenstände aus unterschiedlichen gesellschaftlichen Bereichen aufgegriffen und mit touristischen Semantiken beschrieben werden. Dadurch wechseln sie gewissermaßen das Relevanzsystem, werden neu gerahmt und dadurch zu touristischen Gegenständen.

Entstanden ist der Tourismus durch die Popularisierung des Reisens, die aus dem Ortswechsel zum Zwecke erlebnisförmiger Erholung einen Teil der modernen Massenkultur gemacht hat (Enzensberger 1979; Hachtmann 2010; Makropolus 2008). Dabei muss man nicht nur an die Pauschalreisen denken, zu deren Markenzeichen die Bereitstellung und Standardisierung touristischer Infrastruktur für möglichst viele Nutzer:innen gehört, denn auch der sogenannte Individualtourismus ist zu einem massenkulturellen Phänomen geworden. So hebt Pott hervor, dass der Tourismus damit auf die Folgen reagiere, die die spezifische Inklusionsstruktur moderner Gesellschaften für die Individuen habe (Pott 2011, S. 259). Gemeint ist damit die immer nur selektive, aber zahlreiche Inklusion in verschiedenste Teilsysteme, die Identitätsprobleme ebenso mit sich bringe wie die ,Tendenz der Überinanspruchnahme durch Leistungsrollen“ - inklusive ihrer „Abstraktions- und Selbstdisziplinierungsanforderungen“ (ebd.). Demgegenüber adressiere der Tourismus die Individuen nicht in ihrer Eigenschaft als Rollenträger, sondern in ihrer Ganzheit, das heißt in ihrem persönlichen, körperlichen Erleben und Wahrnehmen (ebd.). Damit biete er Möglichkeiten der in Hinblick auf die gelingende alltägliche Multiinklusion notwendigen Identitätsarbeit, so Pott (ebd. S. 260). Voraussetzung dafür seien die während eines Urlaubsaufenthalts möglichen Strukturlockerungen, das heißt Lockerungen und Va-

\footnotetext{
5 Ob man in Anbetracht eines eigenen Codes und eigener Organisationen nicht doch von einem eigenständigen System sprechen kann, ist unseres Erachtens noch nicht abschließend geklärt.
} 
riation „der alltäglichen Inklusions- und Erwartungsstrukturen“ (ebd. S. 261), die den Urlaub als das Andere des Alltags mit seinen Anforderungen erlebbar werden lassen (Hennig 1999; Sloterdijk 2017). ${ }^{6}$ Durch die Distanz zum Alltag erhält Urlaub immer einen außeralltäglichen Charakter. Mit Tourismus hat man es aber erst dann zu tun, wenn ein Ortswechsel zum Zwecke der Erholung stattfindet. Wir kommen darauf zurück.

Wir wollen im Folgenden zeigen, dass auch Religion ein relevanter Bezugspunkt für den Tourismus ist. Daher untersuchen wir, ob und auf welche Weise Religiöses durch Tourismus einer Recodierung unterzogen wird und damit in den Fokus touristischer Aktivitäten rückt. Allerdings soll das Verhältnis von Tourismus und Religion auch in entgegengesetzter Richtung in den Blick genommen werden. Dies bedeutet, der Frage nachzugehen, inwiefern Religion den Tourismus für religiöse Kommunikation nutzt, sich also religiöse Recodierungen des Tourismus beobachten lassen. Dafür konzentrieren wir uns im Folgenden vor allem auf die kommunikative Seite sowohl von Tourismus als auch von Religion.

\subsection{Religiöse und touristische Kommunikation}

Religion und Tourismus untersuchen wir anhand ihrer Ausdrucksformen als religiöse und touristische Kommunikation. Unser Verständnis von Kommunikation ist dabei nicht auf sprachliche bzw. schriftliche Formen begrenzt, sondern schließt Artefakte, Praktiken usw. ein (Knoblauch 2005, 2009). Aufgrund des explorativen Charakters beschränken wir uns in diesem Aufsatz jedoch vor allem auf sprachliche Kommunikation, wie sie beispielsweise auf Internetseiten oder Flyern zu finden ist. In einem ersten Schritt werden zunächst religiöse und touristische Kommunikation voneinander unterschieden und ihre jeweiligen Besonderheiten herausgestellt. Auf diese Weise wird es möglich, bei der anschließenden Interpretation des empirischen Materials wechselseitige Bezüge, Anleihen, Überformungen und die Überführung in andere Relevanzsysteme zu identifizieren.

\subsubsection{Touristische Kommunikation}

Zentraler Topos touristischer Kommunikation ist wie oben schon erwähnt erlebnisförmige Erholung. Damit reagiert Tourismus auf das Problem der Abwesenheit von Individualität und Körperlichkeit im Rahmen der rollenförmigen Inklusion von Individuen in die gesellschaftlichen Teilsysteme und ihre Anforderungsstrukturen. Demgegenüber stellt er Strukturvarianz und Möglichkeiten der Identitätsarbeit bereit (Pott 2011, S. 260). Touristische Kommunikation verspreche und organisiere einen

\footnotetext{
6 Die ab Ende des 19. Jahrhunderts in zunehmender Zahl entstehenden Fremdenverkehrsvereine und Reiseveranstalter ,erkennen auf ihre je eigene Weise das historisch neue Umweltproblem der modernen Gesellschaft (...) und richten sich daran aus. Mit ihren jeweiligen Angeboten und Werbeaktionen reagieren sie auch auf bereits existente touristische Mobilität, die sie als Ausdruck eines entsprechenden Bedürfnisses nach Alltagsdistanz interpretieren. Auf diese Weise definieren, stabilisieren und stimulieren Organisationen zugleich die Nachfrage, auf die sie reagieren“ (Pott 2011, S. 262). Zwischen modernen Bedürfnissen nach Strukturlockerung und touristischen Organisationen bestehe daher ein wechselseitiges Steigerungsverhältnis, so Pott.
} 
solchen vorübergehenden „Ausstieg“ aus dem Alltag, der immer auch die vorübergehende „Suspendierung“ der normalen Rollenbeziehungen bedeute, so Pott (ebd.) in Anlehnung an ältere freizeitsoziologische Arbeiten von Erwin K. Scheuch (1969).

Da der Code „erlebnisförmige Erholung“ sehr allgemein gehalten ist, lassen sich im Tourismus Zweitcodierungen als systeminterne Ausdifferenzierungen des Codes beobachten, durch die Komplexität reduziert werden kann (vgl. dazu Luhmann 1987b, S. 239ff.). Zweitcodierungen erleichtern Selektionen und tragen zur Herstellung von Anschlussfähigkeit bei. Beispiele für solche Zweitcodierungen sind z.B. sehenswert/nicht sehenswert oder auch authentisch/nicht authentisch (zu letzterer vgl. Schäfer 2015). Dementsprechend kann touristische Kommunikation unterschiedlich akzentuiert sein: Entspannung, Vergnügen, Entdeckung oder auch Abenteuer sind dabei sicherlich nur einige der möglichen konkreten Fassungen (Pott 2011, S. 258). Der Tourismus macht dazu verschiedene Angebote, die auf Erwartungen von Reisenden reagieren, diese aber auch erst herstellen und festigen. Dabei geht es immer um das Versprechen des Besonderen, sich vom Alltag Unterscheidenden und damit die verbundenen Möglichkeiten der nicht-alltäglichen Differenzerfahrung (ebd., vgl. auch Pechlaner und Volgger 2017, S. 4). Touristische Kommunikation korrespondiert insofern mit zahlreichen sekundären Unterscheidungen, wie die zwischen Arbeit und Freizeit, Pflicht und Freiheit, Heimat und Fremde, Routine und Ausnahme, Lästigem und Erstrebenswertem. Sie findet an unterschiedlichen touristisch markierten Orten und institutionellen Settings statt. Es handelt sich zum einen um nah am Alltag befindliche Orte wie lokale Reisebüros, Messen, Bahnhöfe oder Ausstatter, des Weiteren lässt sie sich aber natürlich auch an den bereisten Orten selbst finden: in Hotels, Bars, örtlichen Informationsbüros etc. Dabei kommen diverse Medien zum Einsatz: Reiseführer, Reiseberichte in Printmedien oder Filme über touristische Destinationen, Werbematerialien von Reiseveranstaltern und touristischen Dienstleistern, Schilder (Sieger und Bendzulla 2018), Internetauftritte, Audioguides bis hin zu Apps und vieles mehr.

Touristische Kommunikation zeichnet sich darüber hinaus wesentlich dadurch aus, dass sie bestimmt, was sehens- und erlebenswert ist. Die durch den Ortswechsel ermöglichte und angestrebte Differenzerfahrung bedarf einer kommunikativen Begleitung, die auf Relevantes, Besonderes, Verlockendes aufmerksam macht. Zivilisationsferne Natur und unberührte Geschichte sind dabei nach wie vor herausragende Leitbilder (Enzensberger 1979, S. 190). Indem Orte und Dinge mit einer spezifischen Semantik des Erlebenswerten verknüpft werden (Sehenswürdigkeiten), wirken Tourismus und touristische Kommunikation raumbildend. Dies wird im Kapitel 2.3. noch einmal aufgegriffen.

\subsubsection{Religiöse Kommunikation}

Ebenso wie beim Tourismus lässt sich auch für Religion ein Bezugsproblem identifizieren, das mit Hilfe einer spezifischen Semantik bearbeitet wird. Demnach bearbeitet religiöse Kommunikation Probleme im Spannungsfeld von Kontingenz und Konsistenz, von Unordnung und Ordnung, von Tradition und Erneuerung mit Hilfe eines speziellen Codes, nämlich mit der Unterscheidung von Immanenz und Transzendenz (Luhmann 1987b, 1998; Pollack 1995; Krech 2011, S. 33 f.). Transzendenz 
kann dabei unterschiedliche Reichweiten haben; systematisch geht es um die Unterscheidung von Diesseits und Jenseits, von Bestimmtem und Unbestimmtem, von Verfügbarem und Unverfügbarem. Religiöse Kommunikation impliziert immer eine Festlegung, nämlich darauf, dass das, was kommuniziert wird, auch geglaubt wird. Dem Reden muss demnach ein inneres Erleben entsprechen. Luhmann hat dies für die „,moderne Individuen-Religion“ konstatiert: Für sie sei „ein (expliziter oder impliziter) Hinweis auf eine korrespondierende innere Einstellung unerlässlich. Religiöse Kommunikation wird nur als Ausdruck einer ihr entsprechenden Auffassung anerkannt“" (Luhmann 1998, S. 144 f.). Gefordert und unterstellt ist also Aufrichtigkeit und Authentizität.

Religiöse Kommunikation findet vor allem in religiös markierten Kontexten, wie z. B. Kirchen- oder Moscheegemeinden oder anderen institutionell gerahmten Situationen, statt. Sie kann vorrangig sprachlich oder nicht-sprachlich sein. Sprachliche Formen sind z.B. Bekenntnisse, Predigten, Gebete usw.; sie greifen oft religiöse Überlieferungen auf, geben sie in Erzählungen wieder und beziehen sie auf konkrete (gegenwärtige) Situationen. Dabei finden (Um-)Deutungen und Übersetzungen statt. Nicht-sprachliche religiöse Kommunikation greift auf bildliche und symbolische Formen zurück. So werden in der Bibel geschilderte Szenen oder Ereignisfolgen in Bildern oder Bilderfolgen wiedergegeben, oder es werden religiöse Symbole aufgegriffen und weiter bearbeitet. Dabei können sich bildliche, performative und sprachliche Elemente ergänzen.

Von religiöser Kommunikation unterscheiden wir Kommunikation über Religion (Sammet 2006). Das schließt an Luhmanns Unterscheidung zwischen einer Kommunikation mit Gott und der Kommunikation über Gott an (vgl. Luhmann 1987a, S. 232). Kommunikation über Gott ist demnach dadurch gekennzeichnet, dass sie eine Leugnung der Existenz Gottes, den Streit über seinen Namen und seine Attribute, eine Kritik an seiner Schöpfungsleistung und Diskussionen über Gottesbeweise zulasse. Kommunikation mit Gott müsse dagegen seine Existenz unterstellen. Um Kommunikation über Religion geht es daran anschließend, wenn keine Festlegungen stattfinden, sondern z.B. Negationen, Alternativformulierungen, Abwägungen und Distanznahmen möglich und zu beobachten sind. ${ }^{7}$ Kommunikation über Religion kann auch mit Vergleichen operieren und erfolgt von einem Standpunkt außerhalb der Religion. Solch ein Wechsel von religiöser Kommunikation zu Kommunikation über Religion lässt sich - so unsere These - in touristischen Dokumenten beobachten.

Eine Bedingung für Kommunikation über Religion ist ein gesellschaftlicher Kontext, der Reflexivität begünstigt und in dem Wissen über verschiedene Religionen und verschiedene Zeiten verfügbar ist. Das ist in modernen Gesellschaften verstärkt der Fall. Ein weiteres Merkmal moderner Gesellschaften befördert ebenfalls Kommunikation über Religion, nämlich ihre funktionale Differenzierung. Kommunikation über Religion kann Religion von außen, also systemextern beobachten, z. B. in

\footnotetext{
7 „Würde die Gesellschaft sich auf Kommunikation über Gott beschränken, würde sie Negationsmöglichkeiten und Alternativformulierungen Tür und Tor öffnen. (...) In der Kommunikation mit Gott ist dagegen all diese Kontingenz ausgeschlossen: Man könnte und würde ja nicht mit ihm kommunizieren, wenn es ihn nicht gäbe“ (Luhmann 1987a, S. 232).
} 
der Politik, der Wissenschaft oder der Kunst. Religion wird dann zum Gegenstand politischer Debatten, wissenschaftlicher Analysen oder auch ästhetischer Erfahrung. Wir werden dies am Beispiel eines weiteren Bereichs moderner Gesellschaften zeigen, und zwar am Tourismus. Religion wird im Tourismus als etwas recodiert, das erlebnisförmige Erholung verspricht.

\subsection{Die Produktion von Räumen durch Tourismus am Beispiel des Wanderns}

Die konstruktivistische Wende in der Raumsoziologie, wie sie hierzulande vor allem durch die Arbeiten Martina Löws (2001) angestoßen wurden, hat darauf aufmerksam gemacht, dass Räume nicht als objektiv gegebene Territorien zu begreifen sind. Sie entstehen vielmehr erst durch Prozesse der (An-)Ordnung bzw. Platzierung von Lebewesen und Dingen resp. Gütern an Orten (spacing) und deren Verknüpfung über Wahrnehmungs-, Vorstellungs- oder Erinnerungsprozesse durch die beteiligten Akteure (Synthese).

Vor diesem Hintergrund muss der Tourismus als einer der großen Agenten von Raumproduktion begriffen werden. Da für touristisches Erleben die Bewegung hin zum touristischen Ort zentral ist, kommt der Mobilität als Bewegung im Raum eine zentrale Bedeutung zu. Denn der Tourismus benötigt spezifische Orte, die zu touristischen Destinationen gemacht werden können, indem sie mit touristischer Semantik beschrieben werden. Darüber hinaus gibt es Formen des Tourismus, in denen das touristische Erleben direkt mit der Bewegung im Raum verbunden ist, z. B. bei Rundreisen, Stadtspaziergängen und Führungen oder Wander- und Radtouren. Insofern muss man wohl sagen, dass im Falle des Tourismus raumbezogene Unterscheidungen wie ,hier - dort“, ,nah - fern“ oder auch ,regional - global“" nicht nur funktional sind, sondern dass ihnen eine geradezu konstitutive Bedeutung zukommt. Der Tourismus lebt vom Ortswechsel und dem damit verknüpften Versprechen erlebnisförmiger Erholung. Dabei begleitet touristische Kommunikation nicht nur den Ortswechsel und die mit ihm einhergehende Erwartung von Strukturlockerung, sie greift auch maßgeblich in die Wahrnehmung und Deutung des vorübergehenden Domizils durch die Reisenden ein. Was auf diese Weise entsteht, ist eine ,tourismusspezifische Raumordnung“ (Pott 2011, S. 263). Regionen, konkrete Orte, Artefakte und Dinge werden einer spezifischen Interpretation unterzogen, die auf das jeweils Besondere, Sehenswürdige, Außeralltägliche zielt: Hier solle man sich aufhalten, dieses oder jenes besichtigen, etwas durchqueren, überwinden oder verweilen (Pott 2011, S. 256). Diese Synthese erzeugt auf Seiten der Reisenden Erwartungen, die die Aneignung des touristischen Raumes maßgeblich strukturieren und lenken.

Neben den geographischen Eigenarten oder historischen Besonderheiten (Enzensberger 1979) unterzieht touristische Kommunikation auch Elemente verschiedener gesellschaftlicher Teilsysteme einer Recodierung. Dem Tourismus als sozialem Strukturzusammenhang haftet dabei etwas Parasitäres an. Da er ständig spezifische Orte benötigt, die zu touristischen Destinationen gemacht werden können, ist er in starkem Maße umweltabhängig und auf die Gegebenheiten gesellschaftlicher Teilbereiche angewiesen. So werden beispielsweise eine alte Zeche (Wirtschaft) als sehenswürdig, ein Kunstmuseum (Kunst) als interessant oder ein Tauchkurs (Sport) als unvergesslich etc. angepriesen und ihr Besuch damit als erlebenswert und erhol- 
sam markiert. Dabei treten diese Aspekte häufig in Kombination auf: Beispielsweise erfolgt der Hinweis auf die stillgelegte Zeche verknüpft mit dem Verweis auf das Ruhrgebiet als einer besonderen, industriekulturell überformten Landschaft. In anderen Fällen wird man den Hinweis auf ein Museum in Verbindung mit Informationen über die historische Bedeutsamkeit bestimmter Ausstellungsstücke finden usw.

Wir wenden uns im Folgenden vor allem solchen Formen touristischer Kommunikation zu, die in Gestalt des Wanderns Bewegung im Raum fokussieren und dabei Bezug auf Religiöses nehmen. Seit einiger Zeit erfreut sich das Wandern wieder einer zunehmenden Beliebtheit. ${ }^{8}$ Touristische Anbieter organisieren dementsprechende Angebote oder stellen vor Ort die nötigen Infrastrukturen und Rahmenbedingungen bereit. Dazu gehört vor allem die Ausweisung von Wegen und Routen. Damit wird einerseits garantiert, dass die relevanten touristischen Orte (die „Sehenswürdigkeiten") angemessene Aufmerksamkeit erfahren. Andererseits wird die Bewegung im Raum selbst als touristisches Erleben konzipiert. Bei der Ausweisung von Wanderwegen erhalten örtliche Tourismusagenturen Unterstützung vom „Deutschen Wanderinstitut" ${ }^{\text {"9 }}$, das sich der Erforschung des Wanderns und der Zertifizierung von „Premiumwanderwegen“ widmet: Dies soll zur besseren und gezielteren Vermarktung von Wanderwegen beitragen, indem ,Höchste Erlebnisqualität und gezielte Markenarbeit“" miteinander verbunden werden. Die zertifizierten „Premiumwanderwege“ sollen sich durch ,besondere Ziele und viele Höhepunkte“ auszeichnen. Das können ,Aussichten, Waldbilder, Gewässer, Felsstrukturen, kulturhistorische Kleinode und vieles mehr“ sein. In Summe tragen sie zu ,,abwechslungsreichen Wanderungen bei“" - so die Argumentation (ebd.). ${ }^{10}$ In diesem Zusammenhang finden sich auch Wanderwege, zu deren Besonderheit religiöse Bezugnahmen gehören. Diese werden nun am Beispiel von Wanderwegen in Süddeutschland eingehender untersucht.

\section{Methodisches Vorgehen}

Die Untersuchung trägt einen explorativen und insofern vorläufigen Charakter. Das zugrunde liegende Datenmaterial beschreibt bzw. bewirbt Wanderwege, für die religiöse Bezugnahmen zentral sind, z.B. Kapellenwanderführer, Broschüren von kirchlichen Tourismusbeauftragten oder örtlichen Tourismusbüros, Internetauftritte

\footnotetext{
8 Anzeiger dieser neuen Popularität sind Bücher wie das von Manuel Andrack (2011): „Das neue Wandern: Unterwegs auf der Suche nach dem Glück“.

9 „Das Deutsche Wanderinstitut ist ein Zusammenschluss von Wanderexperten, die ständig an der Entwicklung von Qualitätsstandards für alles, was mit dem Wandern zu tun hat, arbeiten. Grundlage für diese Arbeit ist wissenschaftliche Forschung. Wir wollen das Wandern als Naturerfahrung und touristisches Angebot unter Berücksichtigung des Naturschutzes fördern und dem Tourismus die entsprechenden Marketinginstrumente zur Verfügung stellen." https://www.wanderinstitut.de/deutsches-wanderinstitut/ (Abruf 5. Februar 2021).

10 https://www.wanderinstitut.de/deutsches-wanderinstitut/premiumwandern/zertifikate/ (Abruf 5. Februar 2021). Allerdings kann nicht eingeschätzt werden, wie viele Menschen diese Wanderwege tatsächlich nutzen, denn die Wanderungen werden zumeist - außer im Falle von Führungen - individuell und unorganisiert unternommen. In manchen Urlaubsregionen sind Wanderwege Teil einer touristischen Infrastruktur mit ganz unterschiedlichen Erholungs- und Erlebnismöglichkeiten.
} 
(bspw. zu Pilgerwegen), Zeitungsartikel u.v.m. Es handelt sich um Material, das touristische Kommunikation dokumentiert, da es im untersuchten Feld von touristischen Akteuren selbst erzeugt wurde. Es stellt damit gewissermaßen ,naturwüchsige (...) protokollierte Wirklichkeit" dar (Oevermann 2000, S. 87). ${ }^{11}$ Gesammelt und recherchiert wurde das Datenmaterial in verschiedenen Urlaubsregionen (z.B. in lokalen Touristeninformationszentren oder Auslagen in Kirchen) sowie im Internet. Als touristisches Informations- und Werbematerial enthalten die Dokumente sowohl verbale als auch bildliche Elemente, wie z. B. Fotos, graphische Darstellungen der Wegeführung, Logos und Ähnliches.

Für diesen Beitrag wurde Datenmaterial zu Wanderwegen im Schwarzwald ausgewählt, da sich in ihm unterschiedliche touristische Aneignungsweisen auf exemplarische Weise dokumentieren. Darüber hinaus eröffnet die Beschränkung auf einen lokal begrenzten Kontext die Möglichkeit späterer Vergleiche. In Hinblick auf den lokalen Kontext wurden die untersuchten Fälle also zunächst mit einer minimalen Kontrastierung gesampelt. In Hinblick auf die Generalisierung wurde theoretisch gesampelt, d.h. es wurden kontrastierende Fälle gesucht, die das Typenfeld anreichern und so nach und nach zu einer theoretischen Sättigung führen (können) (Strauss 1991; Strauss und Corbin 1996). Zu betonen ist, dass die jeweiligen Wanderwege oder touristischen Daten (z.B. Flyer, Wanderwegdarstellungen usw.) für unsere Analysen nicht als solche von Interesse sind, sondern die in ihnen zum Ausdruck kommenden kommunikativen Strategien touristischer und religiöser Kommunikation, die im Ergebnis zu einer Touristifizierung von Religion bzw. umgekehrt zu einer spirituellen Aufladung des Tourismus führen. So können anhand eines Dokuments theoretisch auch mehrere Strategien rekonstruiert werden.

Die Auswertung der sprachlichen Elemente erfolgte feinanalytisch nach dem Verfahren der Objektiven Hermeneutik (Oevermann 1983, 2000; Sammet und Erhard 2018). In der folgenden Präsentation der Fallanalysen werden die graphische Gestaltung der Dokumente mit der Platzierung der bildlichen und sprachlichen Elemente kurz skizziert. Die Rekonstruktion von Fallstrukturen geht jedoch maßgeblich von der Analyse der sprachlichen Elemente aus, daher wird in der Darstellung der Ergebnisse der Schwerpunkt auf diese Passagen gelegt.

\section{Religion und Tourismus - drei Verhältnisse}

Nimmt Tourismus auf gesellschaftliche Teilbereiche Bezug, unterzieht er sie typischerweise einer Recodierung. In den folgenden Fallanalysen wollen wir nun genauer rekonstruieren, wie eine solche Recodierung im Falle von Religion aussehen kann, und dadurch unterschiedliche Spielarten einer solchen Recodierung herausarbeiten. So rahmt Tourismus Religion in manchen Fällen als kulturelles Erbe (4.1).

\footnotetext{
11 Oevermann unterscheidet inszenierte von naturwüchsiger protokollierter Wirklichkeit danach, ,ob die Protokolle, die wir analysieren, ausschließlich zu diesem Zweck angefertigt wurden oder ob die protokollierte Praxis selbst, unabhängig von der Datensammlung zu Forschungszwecken, das der Sequenzanalyse zugrunde liegende Protokoll ohnehin hinterlassen hätte“ (Oevermann 2000, S. 87 f.; Herv.i.O.). Als für die Forschung inszenierte Protokolle sind demnach z. B. Interview- oder Gesprächstranskripte, Beobachtungsprotokolle usw. zu bezeichnen.
} 
Eine zweite Spielart nutzt den religiösen Kontext als verkaufsfördernde Kulisse für das eigentlich im Vordergrund stehende kulinarische, wissensvermittelnde oder gesundheitliche Angebot (4.2). Bei diesen beiden Strategien handelt es sich um Formen von Kommunikation über Religion. Eine dritte Variante überschreitet die damit einhergehende Distanz und lässt sich als eine genuin religiöse Kommunikation charakterisieren. Bezeichnenderweise handelt es sich dabei nicht um eine touristische Recodierung von Religion, sondern - umgekehrt - um eine religiöse Recodierung des Tourismus, die durch eine spirituelle Aufladung von Landschaft gekennzeichnet ist (4.3). Auf diese Weise wird touristisches Erleben anschlussfähig gemacht für religiöse Kommunikation und damit in ein religiöses Referenzsystem überführt. Im Folgenden werden wir diese Formen der Touristifizierung von Religion näher erläutern und anhand von Fallmaterial herausarbeiten. Dabei handelt es sich um Informationsmaterialien zum „Klosterpfad“ zwischen Bad Herrenalb und Frauenalb im Nordschwarzwald und zu Kapellenwanderwegen im Hochschwarzwald. ${ }^{12}$

\subsection{Musealisierung von Religion - Vergegenwärtigung des Vergangenen}

Religiöse Gebäude und Artefakte waren lange Zeit vor allem Ziel von Pilgerreisen und Wallfahrten von Gläubigen. Mit dem Aufkommen und der Verbreitung bürgerlicher Bildungsreisen, aber auch durch das in zunehmendem Maße verfügbare kunstund architekturhistorische Wissen wurden religiöse Gebäude und Artefakte immer mehr zum Ziel oder Bestandteil profaner Reisen. Damit einher ging die Kultivierung eines historisierenden und ästhetisierenden Blicks, der das Religiöse in gewisser Weise auch auf Abstand bringt (Karstein 2021). Die in vielen religiösen Gebäuden mittlerweile obligatorisch zu findenden Informationstafeln und Broschüren zur Baugeschichte oder kunsthistorischen Besonderheiten tragen dem Rechnung. Aber auch thematische Wanderwege werden zunehmend um religiöse Gebäude herum gestaltet. Mit welchen Deutungen und Recodierungen des Religiösen sie beworben werden, soll am Beispiel des „Klosterpfades“ zwischen Bad Herrenalb und Frauenalb im Nordschwarzwald herausgearbeitet werden. Die folgende Auswertung setzt mit den einleitenden Passagen zur Detail-Präsentation des Klosterpfads im Internet ein (Abb. 1).

In der Überschrift wird in elliptischer Form eine zeitliche (1000 Jahre) mit einer zweidimensionalen räumlichen $(5000 \mathrm{~m})$ Bestimmung verbunden. Eine solche verkürzte Information ist typisch für eine Überschrift; sie macht neugierig und lässt erwarten, dass im Folgenden diese Verbindung inhaltlich ausgefüllt wird. Insofern implizieren beide Angaben eine Verdichtung und fordern Spezifizierung heraus. Die Autor:innen des Textes können dabei schon mit spezifischen Erwartungen der Leser:innen rechnen, denn die Webseite wird nicht zufällig gefunden, sondern mit einem konkreten touristischen Interesse an den Orten Bad Herrenalb und Frauenalb und an der touristischen Aktivität Wandern aufgesucht. Dieses Interesse wird durch

\footnotetext{
12 Ein solches Datenmaterial stellt in methodischer Hinsicht einige Herausforderungen, zum einen durch die Nutzung von bildhaften Elementen, wie z. B. Fotos, Logos und graphischen Darstellungen, zum anderen durch die infolge der Faltung komplex strukturierte Sequenzialität. Wir konzentrieren uns in unseren Analysen auf sprachliche Elemente.
} 


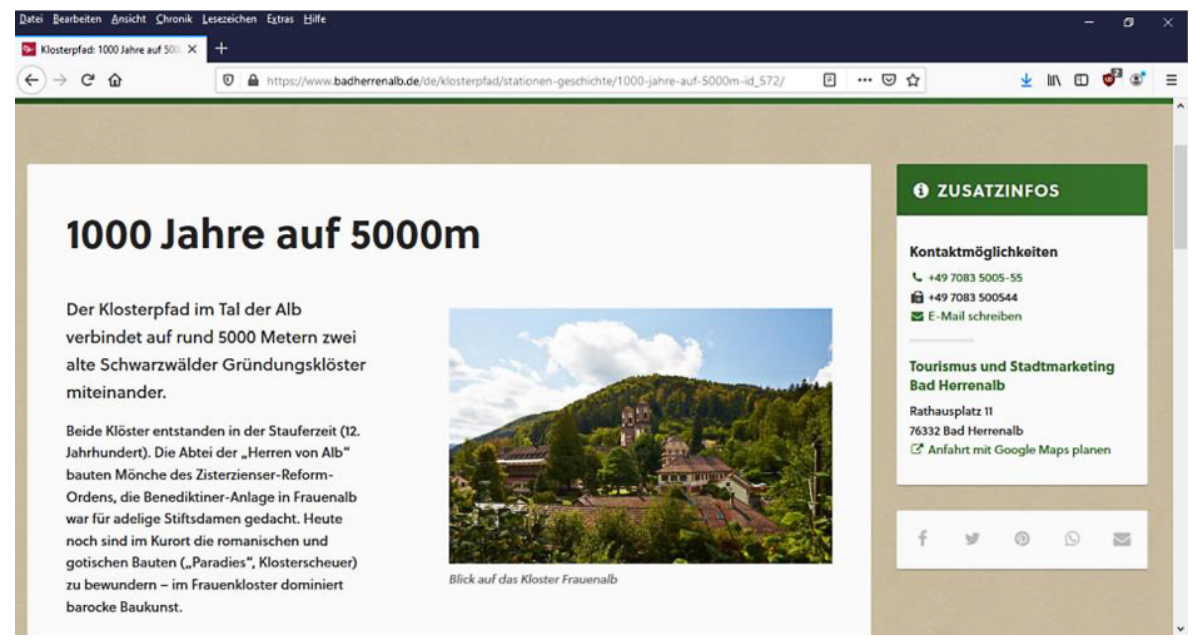

Abb. 1 Der Klosterpfad zwischen Bad Herrenalb und Frauenalb. Screenshot der Webdarstellung (1) (Quelle: https://www.badherrenalb.de/de/klosterpfad/stationen-geschichte/1000-jahre-auf-5000m-id_ 572/ (Abruf 31. Dezember 2020))

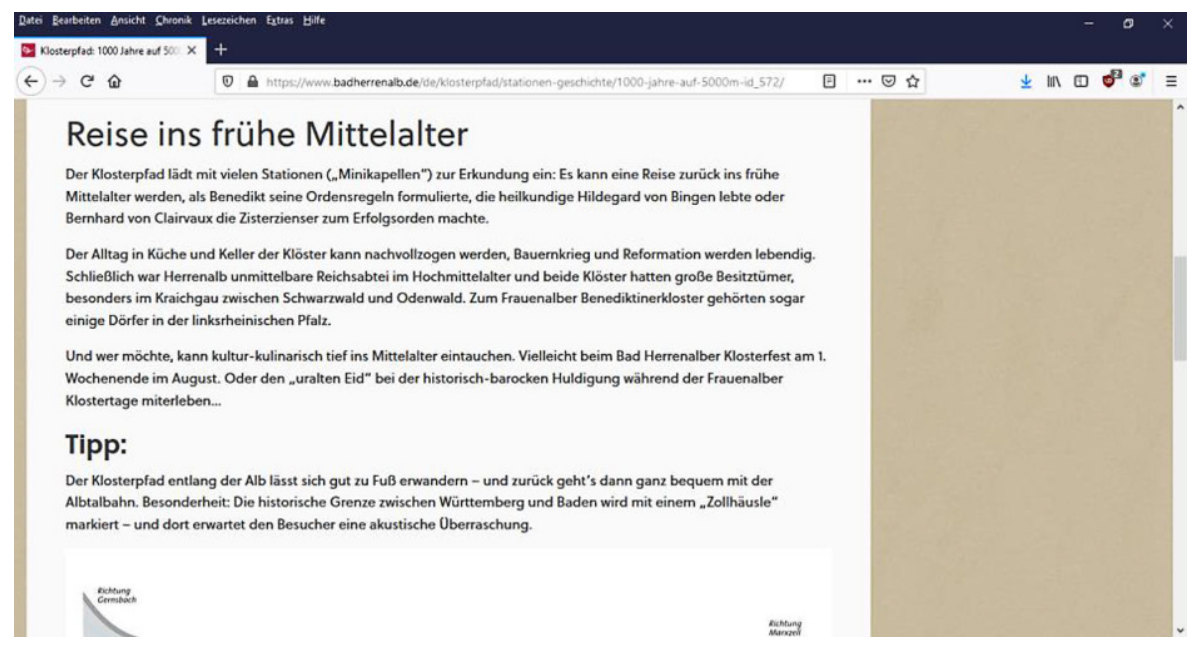

Abb. 2 Der Klosterpfad zwischen Bad Herrenalb und Frauenalb. Screenshot der Webdarstellung (2) (Quelle: https://www.badherrenalb.de/de/klosterpfad/stationen-geschichte/1000-jahre-auf-5000m-id_ 572/ (Abruf 31. Dezember 2020))

den Text auf einen vergleichsweise kurzen Wanderweg und auf kulturhistorische Sehenswürdigkeiten gelenkt: Der Ort wird als ein Flusstal konkretisiert, die Entfernung als Weg zwischen zwei Klöstern, die zugleich für den angekündigten Zeitrahmen von 1000 Jahren stehen. Dieser Zeitraum wird in den weiteren Erläuterungen mit diesen beiden Klöstern verknüpft: Er reicht von der Entstehung „,in der Stauferzeit (12. Jahrhundert)“ - für die „1000 Jahre“ großzügig bemessen sind - bis in die Gegenwart des Kurortes. Dies wird durch die noch bestehenden historischen Gebäude 


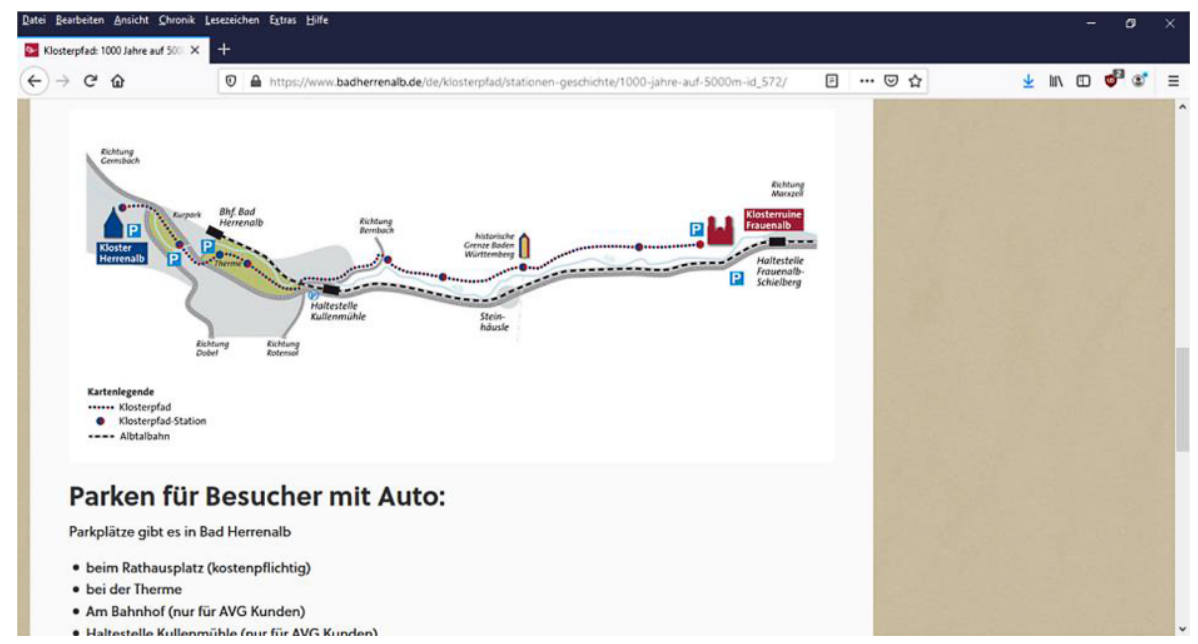

Abb. 3 Der Klosterpfad zwischen Bad Herrenalb und Frauenalb. Screenshot der Webdarstellung (3) (Quelle: https://www.badherrenalb.de/de/klosterpfad/stationen-geschichte/1000-jahre-auf-5000m-id_ 572/ (Abruf 31. Dezember 2020))

und im Dokument durch das Foto vom Kloster Frauenalb bezeugt. Die Gebäude werden damit als etwas Bewundernswertes charakterisiert, was sie zu einer touristisch relevanten Sehenswürdigkeit macht.

Die auf die Einleitung folgende Überschrift „Reise ins frühe Mittelalter“ (Abb. 2) greift die touristische Aktivität der räumlichen Mobilität auf und transformiert sie im Kontext des Klosterpfads zu einer zeitlichen Bewegung. Suggeriert wird, dass die Bewegung im Raum einer Zeitreise gleichkomme, was im Grunde die Einbildungskraft der Besuchenden voraussetzt, sich im Angesicht der Zeugnisse der Vergangenheit dahin zurückversetzen zu können. Durch den Verweis auf berühmte Ordensleute wird diese Zeitreise religiös akzentuiert und der die beiden Orte umfassende und durch den „Klosterpfad“ verbindende Raum als religiöser Raum entworfen.

Die Bewegung auf dem Klosterpfad wird anschließend (Abb. 2) mit Versprechen von Erlebnismöglichkeiten verbunden, durch die vergangene Alltagskultur (,,in Küche und Keller der Klöster") und historische Ereignisse der frühen Neuzeit, die für die Religions- und Konfessionsgeschichte in Deutschland eine zentrale Bedeutung haben, vergegenwärtigt werden können. Diese Versprechen werden räumlich nicht nur auf die Stationen des Klosterpfads bezogen, sondern auch mit der Geschichte der Klöster begründet. Durch ihre „Besitztümer“ war ihnen historisch ein größeres Territorium zugeordnet; damit wird ein verschiedene Landschaften (Kraichgau zwischen Schwarzwald und Odenwald sowie linksrheinische Pfalz) umfassender religiöser Raum skizziert. Die touristische Kommunikation des Erlebnisversprechens wird in diesen Passagen didaktisch akzentuiert: zum einen durch die immer wieder einfließenden historischen Informationen, zum anderen durch die Möglichkeit einer authentischen Vergegenwärtigung des Vergangenen. Diese Authentizität (vgl. Schäfer 2015) wird begründet mit Verweis auf die Verankerung am Ort: Das Vergangene 


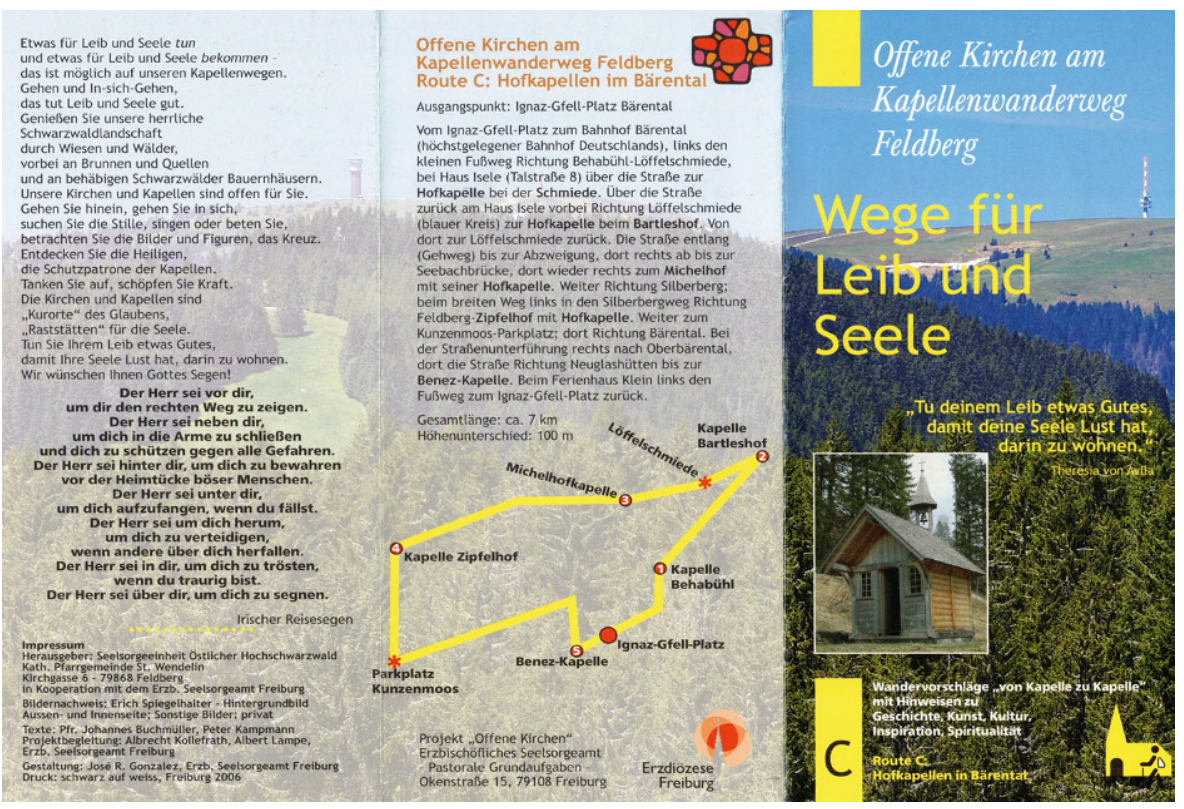

Abb. 4 Informationsblatt Kapellenwanderweg am Feldberg, Route C, Außenseite

ist besonders nah, weil es früher an genau diesem Ort ein Klosterleben gab, von dem heute noch Relikte zu besichtigen sind. Abgeschlossen wird die Passage dementsprechend mit dem Hinweis auf jährliche Höhepunkte der Vergegenwärtigung der religiösen Kultur in Form von Festen. Auf der Webseite finden sich daran anschließend noch eine graphische Darstellung des Wegverlaufs und praktische Hinweise zum Zugang (Abb. 3).

Ein weiteres Beispiel der vergegenwärtigenden Aneignung des Vergangenen findet sich im Faltblatt zu einem Kapellenwanderweg am Feldberg im Hochschwarzwald, der vom Seelsorgeamt der Erzdiözese Freiburg herausgegeben wurde. Diese Präsentation eines Wanderwegs wird demnach von einer kirchlichen Institution verantwortet. Sie trägt den Titel „Offene Kirchen am Kapellenwanderweg Feldberg. Wege für Leib und Seele" und liegt vor Ort in Kirchen und Kapellen aus (Abb. 4). ${ }^{13}$

Auf dem Titelblatt, dessen Hintergrund ein Blick auf den Feldberg bildet und in das ein kleines Foto einer Hofkapelle eingefügt ist, wird das genannte Motto „Leib und Seele“ mit einem Zitat von Theresia von Avila unterlegt: „Tu deinem Leib etwas Gutes, damit deine Seele Lust hat, darin zu wohnen“. Damit wird das Wandern auf dem Kapellenweg nicht nur durch die konkreten Gebäude, sondern auch durch religiöse Kommunikation an Religiöses angeschlossen. Am unteren Ende des Titelblatts stellen die Autor:innen den Inhalt vor: „Wandervorschläge ,von Kapelle zu Kapelle“ mit Hinweisen zu Geschichte, Kunst, Kultur, Inspiration, Spiritualität“.

13 Im Internet ist das Informationsblatt nicht zu finden. Gegenstand der Analyse ist das Faltblatt zur ,Route C: Hofkapellen in Bärental“. 


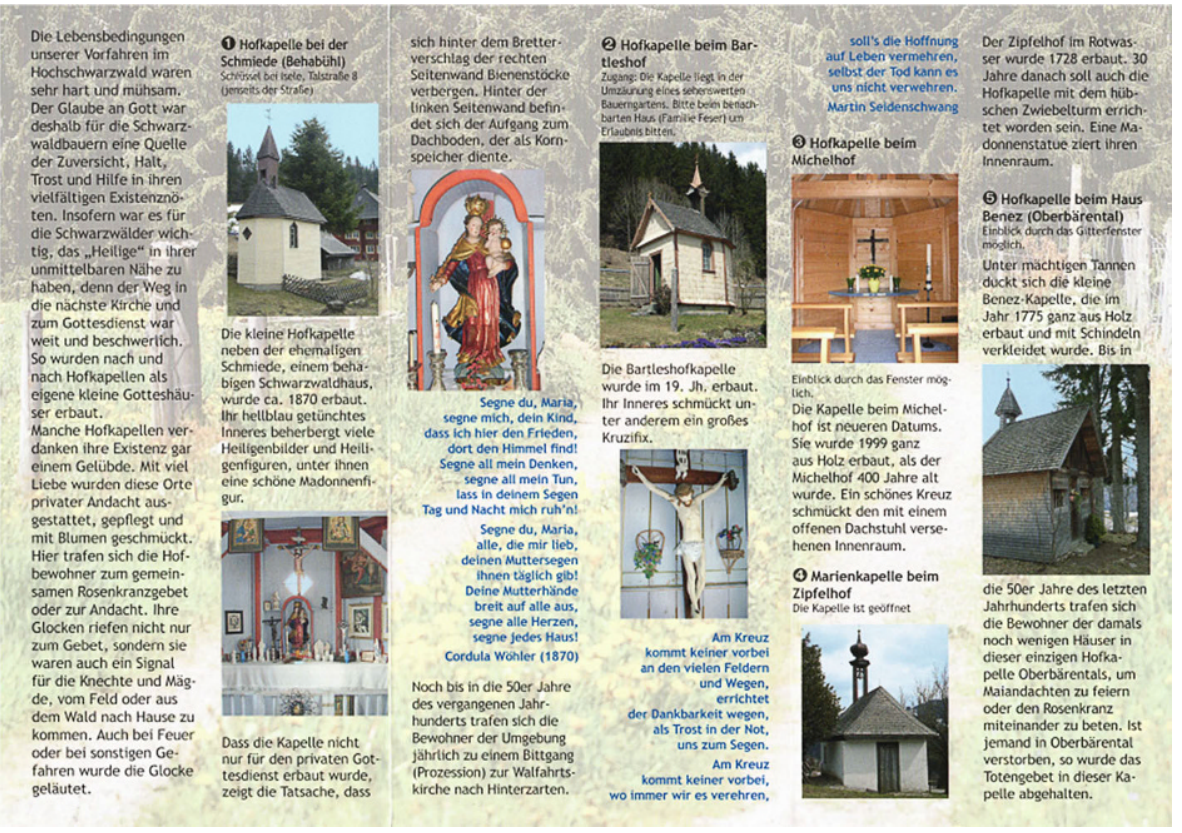

Abb. 5 Informationsblatt Kapellenwanderweg am Feldberg, Route C, Innenseite

Auf der Innenseite werden Fotos von Innen- und Außenansichten der aufzusuchenden Kapellen, Kurzbeschreibungen ihrer Entstehungsgeschichte und ihrer Nutzung ${ }^{14}$ sowie zwei religiöse Gedichte präsentiert (Abb. 5). All dies wäre einer vertieften Ausdeutung wert.

Wir konzentrieren uns an dieser Stelle auf den einleitenden Text des Faltblatts (linke Spalte der Innenseite des Faltblatts):

Die Lebensbedingungen unserer Vorfahren im Hochschwarzwald waren sehr hart und mühsam. Der Glaube an Gott war deshalb für die Schwarzwaldbauern eine Quelle der Zuversicht, Halt, Trost und Hilfe in ihren vielfältigen Existenznöten. Insofern war es für die Schwarzwälder wichtig, das „Heilige“ in ihrer unmittelbaren Nähe zu haben, denn der Weg in die nächste Kirche und zum Gottesdienst war weit und beschwerlich. So wurden nach und nach Hofkapellen als eigene kleine Gotteshäuser erbaut. Manche Hofkapellen verdanken ihre Existenz gar einem Gelübde. Mit viel Liebe wurden diese Orte privater Andacht ausgestattet, gepflegt und mit Blumen geschmückt. Hier trafen sich die Hofbewohner zum gemeinsamen Rosenkranzgebet oder zur Andacht. Ihre Glocken riefen nicht nur zum Gebet, sondern sie waren auch ein Signal für die Knechte und Mägde, vom Feld nach Hause zu kommen. Auch bei Feuer oder bei sonstigen Gefahren wurde die Glocke geläutet.

\footnotetext{
14 Dies wird verbunden mit Hinweisen auf Besonderheiten, z. B. „,eine schöne Madonnenfigur“ oder „,dass
} sich hinter dem Bretterverschlag der rechten Seitenwand Bienenstöcke verbergen“. 
Der erste Satz stellt eine Verbindung zur Vergangenheit her und macht mit dem Pronomen ,unser“ zugleich ein identifikatorisches Angebot an die potenziellen Leser:innen des Faltblattes. Hervorgehoben werden in diesem Satz aber auch die „sehr harten und mühsamen“" Lebensbedingungen des Hochschwarzwaldes. Damit erhält dieser Hinweis eine prominente Stellung im Text. In Bezug auf die Vergangenheit wird Kontinuität und zugleich Distanz hergestellt: Es sind „unsere“ Vorfahren, aber es wird durch die Nutzung des Präteritums ,waren“ auch ausgesagt, dass es heute anders ist. Im touristischen Kontext wird damit vor Augen geführt, dass die besuchte und bewanderte Landschaft von den Vorfahren ganz anders wahrgenommen wurde: Während sie damals als Raum von Arbeit und Not erfahren wurde, ist sie für die touristische Besucherin ein Raum der Erholung und der Freizeit. Die ,vielfältigen Existenznöte“ der Vergangenheit können heute im Modus des touristischen Erlebens vergegenwärtigt und nachempfunden werden, ihnen ist jedoch niemand mehr ausgesetzt. ${ }^{15}$ Im Unterschied zur Darstellung des Klosterpfades wird hier (zunächst) nicht auf religions- und herrschaftsgeschichtliche Aspekte verwiesen, sondern auf die Alltagsgeschichte dieser Region.

Religion kommt im nächsten Schritt ins Spiel, und zwar abgeleitet aus den Lebensbedingungen ${ }^{16}$ der Schwarzwaldbauern. Damit wird ein direkter Zusammenhang zwischen harten Lebensbedingungen und Gläubigkeit unterstellt. Die genannten Hilfeleistungen der Religion - „Zuversicht, Halt, Trost und Hilfe“ - sind insgesamt sehr diesseitig ausgerichtet, da sie auf die Bewältigung der Lebensbedingungen abzielen. Ein transzendenter Horizont ist dabei nicht impliziert, wie es etwa bei der - hier als kontrastierendes Gedankenexperiment eingeführten - Aussage „Das Leben ist ein Jammertal, aber die himmlische Herrlichkeit wartet auf uns" der Fall wäre. ${ }^{17}$ Mit diesem letztlich funktionalen Argument nimmt der Text zur Religion eine distanzierte Perspektive ein. Dieser Eindruck verstärkt sich im folgenden Satz noch einmal, wenn das „Heilige“ in Anführungszeichen gesetzt wird. Mit dieser alltagsweltlichen Funktion und einer weiteren lebenspraktischen Erwägung - das „Heilige“ aufgrund der weiten Wege zur Kirche in der Nähe haben wollen - wird dann der Bau der Hofkapellen erklärt, die Thema und Markierungspunkte des Wan-

\footnotetext{
${ }^{15}$ Hier scheint ein ähnlicher Effekt zu wirken, wie der, den Norbert Elias in Bezug auf Kampfspiele beschrieben hat. Da man durch den Prozess der Zivilisation nur noch selten in reale und physisch bedrohliche Kämpfe verwickelt sei, genieße man spielerische Kämpfe wie die im Sport umso mehr. Auch das Nachempfinden harter Lebensbedingungen kann einem einen wohligen Schauer bescheren durch die Gewissheit, solchen Umständen nicht mehr real ausgesetzt zu sein (Elias 1983).

${ }^{16}$ Es gibt auch Wanderwege im Schwarzwald, die ganz ohne Religionsbezug die Lebensbedingungen in der Vergangenheit thematisieren, wie z. B. der „Hochschwarzwälder Hirtenpfad“. In seiner Beschreibung wird explizit auf die veränderten Lebensbedingungen hingewiesen. „Das bäuerliche Leben auf dem ,hohen Wald" vor 1960 war bescheiden und hart und noch kein Ort, ,wo andere Urlaub machten “". https://www. hochschwarzwald.de/Touren-im-Hochschwarzwald/Geniesserpfad-Hochschwarzwaelder-Hirtenpfad Zugegriffen: 5. Februar 2021.

17 Das ,irdische Jammertal“ ist ein altes religiöses Motiv, das im Sinne der Kompensationsidee dem irdischen Elend eine ewige Seligkeit als „Verheißung“ (Weber 1980, S. 299) mit einer „Vergeltungs- und Erlösungshoffnung“ (300) gegenüberstellt. Im 19. Jahrhundert fungierte das Motiv als Ansatzpunkt von Religionskritik, wie z.B. in Heinrich Heines „Deutschland. Ein Wintermärchen.“ Darin wird vom Lied eines Harfenmädchens erzählt: „Sie sang vom irdischen Jammertal, Von Freuden, die bald zerronnen, Vom Jenseits, wo die Seele schwelgt Verklärt in ew'gen Wonnen. Sie sang das alte Entsagungslied, Das Eiapopeia vom Himmel, Womit man einlullt, wenn es greint, Das Volk, den großen Lümmel.“.
} 
derwegs sind. Wenn die Gläubigkeit der Schwarzwaldbauern mit ihren „harten“ und „mühsamen“ Lebensbedingungen erklärt wird, impliziert das umgekehrt, dass diese Gläubigkeit keinen Grund und Anlass mehr hat, wenn sich die Lebensbedingungen zum Positiven verändern und die Bewältigung des Lebens leichter wird. ${ }^{18}$ Als Folge davon werden die bleibenden Zeugnisse der Gläubigkeit der Vorfahren erklärungsbedürftig, weil sich ihr Sinn und ihre Funktion den heutigen Menschen nicht mehr erschließt. Dem Text scheint diese Annahme implizit zugrunde zu liegen, denn im Weiteren werden die ursprünglichen religiösen, aber auch nicht-religiösen Nutzungen der Hofkapellen erläutert und so den Leser:innen wieder näher gebracht.

In beiden bisher analysierten Wanderwegbeschreibungen wird der bewanderte Raum als ein religiöser Raum der Vergangenheit entworfen. Das vergangene Religiöse ist noch in Spuren und Relikten vorzufinden, die den wandernden Tourist:innen erklärt werden. Die Welt, durch die der Wanderweg führt, wird dadurch zu einem religionsgeschichtlichen Museum gemacht. Daher sprechen wir an dieser Stelle von einer Musealisierung des Religiösen: Religion gehört der Vergangenheit an, sie ist Teil der Kulturgeschichte der bereisten Region. Man hat es hier also mit einer Kommunikation über Religion zu tun, weniger (bzw. nicht nur) mit einer genuin religiösen Kommunikation. Bemerkenswert ist, dass auch religiöse Akteure auf diese Strategie zurückgreifen. Unten (4.3.) werden wir demgegenüber eine Kommunikationsstrategie präsentieren, die das Wandern mit moderner Religiosität verbindet.

\subsection{Kommodifizierung von Religion: Wandern als Konsumerlebnis}

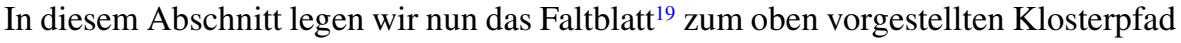
zwischen Bad Herrenalb und Frauenalb zugrunde.

Dieses Faltblatt präsentiert sich den Nutzer:innen mit zwei Außenseiten (Abb. 6), die beide als Titelblatt wahrgenommen werden können. Auf ihnen sind - jeweils umrahmt von Logos und der Nennung der Namen von Projektträgern und ihren Unterstützern (Land Baden-Württemberg, EU) - die gezeichneten Darstellungen eines Mönchs (blau eingefärbt) und einer Nonne (in rot) mit weiß gezeichneten Skizzen von Klostergebäuden vor grauen Hintergrund zu sehen. Zusammen mit dem groß gedruckten Titel „Klosterpfad“ und den in jeweils unterschiedlicher Reihenfolge angeordneten Ortsnamen Bad Herrenalb und Frauenalb wird damit die religiöse Referenz des touristischen Angebots auf den ersten Blick deutlich. So erkennt die Besucherin beispielsweise des Tourismusbüros vor Ort sofort, dass sich der Klosterpfad mit religiösen Themen befasst.

Die Analyse widmet sich im Weiteren dem schriftlichen Text und arbeitet heraus, welche weiteren Kommunikationsstrategien sich in diesem Datenmaterial abweichend zur zuvor rekonstruierten Musealisierung beobachten lassen.

\footnotetext{
18 Dieser Gedanke ist strukturell analog zum ,Security Axiom“ in der religionssoziologischen Forschung. Demnach können reiche Staaten ihren Bewohnern weiter reichenden Schutz vor Risiken und Gefahren und daher größere Sicherheit bieten als arme Staaten, was zum Rückgang der Religion in den reichen Ländern beitrage (vgl. Norris und Inglehart 2004, S. 13 ff.).

19 Das Faltblatt kann auch im Internet heruntergeladen werden: https://www.badherrenalb.de/downloads/? aktuelles=812 Zugegriffen: 22. Januar 2021.
} 


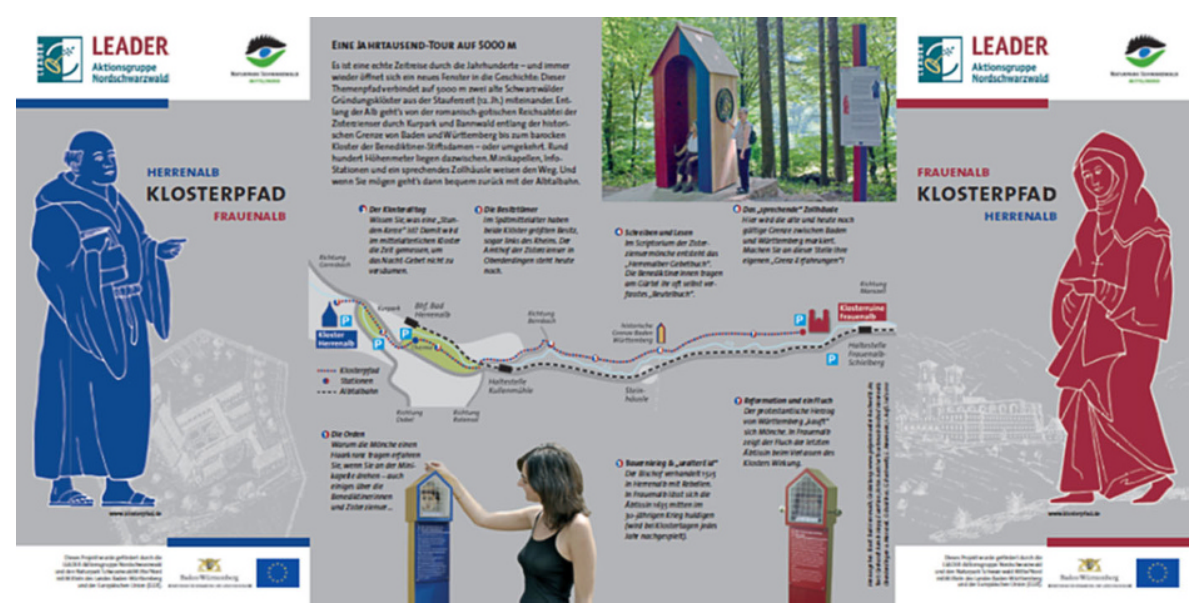

Abb. 6 Informationsflyer zum Klosterpfad von Bad Herrenalb nach Frauenalb (Außenseite)

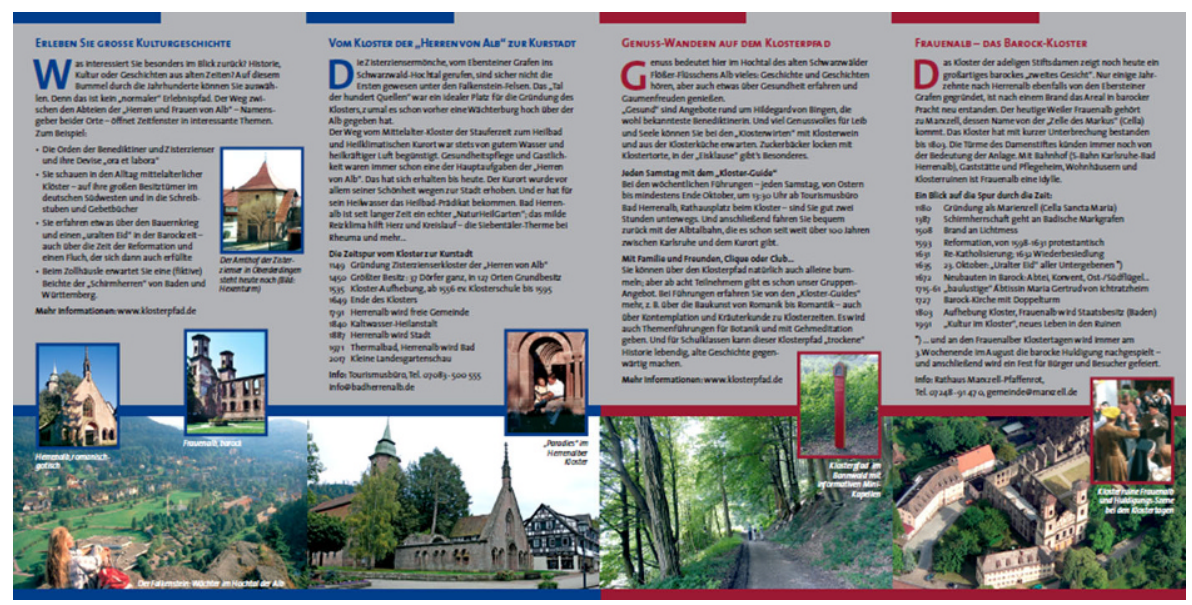

Abb. 7 Informationsflyer zum Klosterpfad von Bad Herrenalb nach Frauenalb (Innenseite)

Die Innenseite des Faltblatts (Abb. 7) ist in vier Teile aufgeteilt. Auf der linken Seite ist der folgende Text zu finden:

\section{ERLEBEN SIE GROSSE KULTURGESCHICHTE}

Was interessiert Sie besonders im Blick zurück? Historie, Kultur oder Geschichten aus alten Zeiten? Auf diesem Bummel durch die Jahrhunderte können Sie auswählen. Denn das ist kein ,normaler“ Erlebnispfad. Der Weg zwischen den Abteien der „Herren und Frauen von Alb“ - Namensgeber beider Orte öffnet Zeitfenster in interessante Themen. Zum Beispiel:

- Die Orden der Benediktiner und Zisterzienser und ihre Devise „ora et labora“

- Sie schauen in den Alltag mittelalterlicher Klöster - auf ihre großen Besitztümer im deutschen Südwesten und in die Schreibstuben und Gebetbücher 
- Sie erfahren etwas über den Bauernkrieg und einen „uralten Eid“ in der Barockzeit - auch über die Zeit der Reformation und einen Fluch, der sich dann auch erfüllte

- Beim Zollhäusle erwartet Sie eine (fiktive) Beichte der „Schirmherren“ von Baden und Württemberg.

Mehr Informationen: www.klosterpfad.de

Der Text ist überschrieben mit einer Aufforderung (,Erleben Sie ...“). Der Begriff „erleben“ entspricht einerseits dem touristischen Code, andererseits knüpft er als Aufforderung an eine Praxis an, die aus der Werbung vertraut ist: „Genießen Sie ...“, „Lassen Sie sich das nicht entgehen ...“, „Schlagen Sie zu ...“, „Entdecken Sie ...“ etc. Darüber hinaus wird durch Großschreibung und die Nutzung eines steigernden (,große“) Adjektivs zusätzlich Aufmerksamkeit erzeugt. Das Angebot, Kulturgeschichte zu erleben, greift auf das Thema des Faltblatts zurück (s. oben), verpackt es aber in anderer Weise. Im Weiteren wird nicht ein Spektrum religionshistorischer Themen aufgemacht, sondern eine Vielfalt von Perspektiven auf das Vergangene, die jedoch stichwortartig-unspezifisch bleiben und gedanklich unterschiedlich ausgefüllt werden können. Während „Historie“ an die die großen Zeitläufte denken lässt, zielen die „Geschichten aus alten Zeiten“ eher auf das Kleine, Konkrete, Partikulare, Anekdotenhafte. „Kultur“ im Kontext des Tourismus kann an Hochkulturelles (wie z. B. die Ausgestaltung von Kirchen durch große Meister oder kirchenmusikalische Veranstaltungen) oder auch an Alltagskulturelles (z. B. Brauchtum oder Lebensweisen) denken lassen. Das Erleben der Kulturgeschichte ${ }^{20}$ wird im Fortgang durch den Verweis auf persönliche Interessen und Wahlmöglichkeiten individualisiert. Da aber auf dem Klosterpfad - wie die Spiegelstriche im Folgenden zeigen - alles angeboten wird, sind alle Möglichkeiten gleichwertig. Damit wird das ,große“ der Kulturgeschichte in viele nebeneinanderstehende Erlebnismöglichkeiten aufgefächert, wobei sich auch wieder inhaltliche Anknüpfungen an das Thema Religion finden (Orden, Klöster, Gebetbücher, Beichte usw.). Der Blick auf die vielfältigen touristischen Angebote wird im Text dann mit einer Freizeitaktivität verbunden, die im Sprachgebrauch mit dem samstäglichen Besuch einer belebten Innenstadt mit ihren Läden und Angeboten verbunden ist: dem „Bummel“. Dieser Begriff bezeichnet einen nicht zielgerichteten, eher zerstreuten und entschleunigten Weg durch die Warenwelt, bei dem der Blick über die ausliegenden Waren schweifen kann und der Kauf nicht durch Notwendigkeit, sondern durch Freude am Genuss motiviert ist. Da die präsentierten Erlebnisangebote thematische Ankerpunkte im Religiösen haben, sprechen wir hier von Kommodifizierung von Religion im Tourismus. An dieser Stelle kann zwar noch davon ausgegangen werden, dass das Erlebnis erwandert werden kann und nicht gekauft werden muss, jedoch zeigt sich beim Weiterlesen, dass das Erleben des Religiösen auch durch Kauf erworben werden kann. In dieser Bezugnahme dient das Religiöse so vor allem als pittoreske und damit verkaufsfördernde Kulisse für

\footnotetext{
${ }^{20}$ Das Versprechen des Erlebens von „Kulturgeschichte“ ist in gewisser Weise paradox, da „Geschichte“ sich konstitutiv auf Vergangenes bezieht, das aufgrund seiner Zeitlichkeit gerade nicht mehr erlebt werden kann.
} 
etwas, das eigentlich im Vordergrund steht, nämlich der Genuss. Dies wird in der zweiten Spalte von rechts weiter ausgeführt:

\section{GENUSS-WANDERN AUF DEM KLOSTERPFAD}

Genuss bedeutet hier im Hochtal des alten Schwarzwälder Flößer-Flüsschens Alb vieles: Geschichte und Geschichten hören, aber auch etwas über Gesundheit erfahren und Gaumenfreuden genießen. „Gesund“ sind Angebote rund um Hildegard von Bingen, die wohl bekannteste Benediktinerin. Und viel Genussvolles für Leib und Seele können Sie bei den „KIosterwirten“ mit Klosterwein und aus der Klosterküche erwarten. Zuckerbäcker locken mit Klostertorte, in der ,Eisklause“ gibt's Besonderes.

Jeden Samstag mit dem „Kloster-Guide“

Bei den wöchentlichen Führungen - jeden Samstag, von Ostern bis mindestens Ende Oktober, um 13:30 Uhr ab Tourismusbüro Bad Herrenalb, Rathausplatz beim Kloster - sind Sie gut zwei Stunden unterwegs. Und anschließend fahren Sie bequem zurück mit der Albtalbahn, die es schon seit weit über 100 Jahren zwischen Karlsruhe und dem Kurort gibt.

Mit Familie und Freunden, Clique oder Club ...

Sie können über den Klosterpfad natürlich auch alleine bummeln; aber ab acht Teilnehmern gibt es schon unser Gruppen-Angebot. Bei Führungen erfahren Sie von den „Kloster-Guides“ mehr, z.B. über die Baukunst von Romanik bis Romantik - auch über Kontemplation und Kräuterkunde zu Klosterzeiten. Es wird auch Themenführungen für Botanik und mit Gehmeditation geben. Und für Schulklassen kann dieser Klosterpfad ,trockene“ Historie lebendig, alte Geschichte gegenwärtig machen.

Mehr Informationen: www.klosterpfad.de

Die angebotenen Waren gehören in verschiedene Kategorien, in denen das Religiöse in verschiedener Weise mit der Bewegung in Raum - also dem Wandern verknüpft wird. Diese Angebote werden im Bereich des Tourismus auch ohne Bezug auf Religion gemacht: erstens das Essen lokaler Spezialitäten, zweitens die Sorge für die Gesundheit und drittens Führungen zur Vermittlung von Wissen über die örtlichen Sehenswürdigkeiten. Während letztere zur Musealisierung des Religiösen beitragen, zielen die zuerst genannten auf den Körper und die Sinne der Reisenden. Gesundheit als Fokus religionsbezogener touristischer Angebote gehen im Text vom Verweis auf Hildegard von Bingen aus und führen zum Angebot von Führungen zu „Kontemplation und Kräuterkunde zu Klosterzeiten“. Die Einkehr in Gasthäusern, die als Rast und Stärkung zu Wanderungen gehören, werden religiös angeschlossen durch gastronomische Begriffe, spezifiziert durch die Verknüpfung mit „Kloster-“ und die Einordnung als „Genussvolles für Leib und Seele“.

Zusammenfassend lässt sich festhalten, dass durch die Semantiken des Wählens und Genießens das Religiöse in verschiedener Form zur Ware gemacht wird, also kommodifiziert wird. Zugleich wird das Religiöse mit Genuss konnotiert und insofern hedonisiert. 


\subsection{Spiritualisierung des Wanderns: Äußere und innere Landschaft}

Im Datenmaterial lassen sich durchaus auch Formen der religiösen Aufladung des Wanderns beobachten. Damit bezeichnen wir die religiöse Recodierung einer touristischen Aktivität, die mit einem Wechsel von touristischer zu genuin religiöser Kommunikation verbunden ist. In der folgenden Darstellung greifen wir auf einen „Kapellenwegführer“ mit dem Titel „Hochschwarzwald hochspirituell“ zurück, der laut Impressum im Jahr 2013 von der Hochschwarzwald Tourismus $\mathrm{GmbH}$ in $\mathrm{Zu}$ sammenarbeit mit einem ökumenischen Redaktionsteam produziert wurde. Er liegt vor Ort z. B. in Kirchen oder Tourismusbüros aus und enthält viele Elemente gebräuchlicher Wanderführer: Wegbeschreibungen mit touristisch interessanten Orten als Wegmarken (hier: Kirchen, Klöster und Kapellen), Skizzen des jeweiligen Höhenprofils, Informationen zur Anfahrt, zu Gelegenheiten zur Einkehr sowie zu weiteren Sehenswürdigkeiten usw. Sie werden ergänzt durch kurze religiöse Texte in kleinen Boxen. Den Darstellungen von insgesamt 27 Wandertouren ist ein „Vorwort“ vorangestellt, das wir im Folgenden analysieren wollen (Abb. 8).

Dieses Vorwort ist durch visuelle und schriftliche Elemente gerahmt. So befindet sich in der Kopfzeile oberhalb des Logos „Kapellenwegführer“ der Begriff „hochspirituell“, der zusammen mit „Hochschwarzwald“ schon auf dem Titel des Buches zu finden ist. Durch das mit Kursivierung abgesetzte Präfix ,hoch“ wird das Adjektiv „spirituell“ einerseits gesteigert und intensiviert, andererseits wird es räumlich kontextualisiert: Es geht um Spiritualität in der Höhe, hier: im Hochschwarzwald. Durch zwei Fotos wird die Spiritualität illustriert und damit assoziativ angereichert: Rechts neben dem Vorwort ist ein Bild(ausschnitt) platziert, auf dem in einer Art Halle an den Wänden und auf Tischen Kruzifixe und Bilder mit re-

Abb. 8 Kapellenwegführer, Vorwort
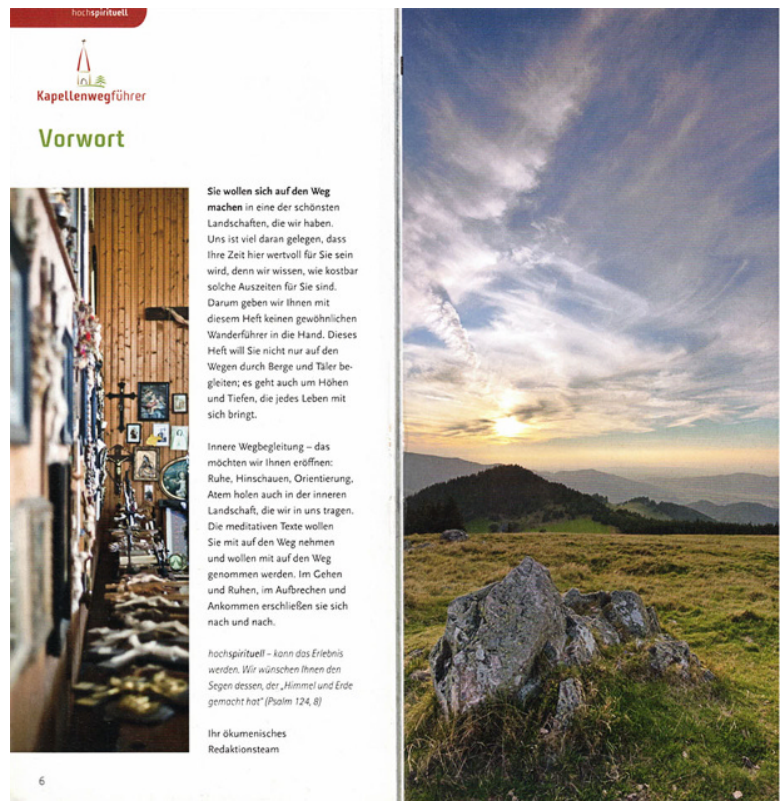
ligiösen Darstellungen ausgestellt sind. Dem Ausschnitt ist nicht zu entnehmen, ob es sich um eine Ausstellung oder eine Verkaufsveranstaltung handelt. Die dem Vorwort gegenüberliegende Seite wird vollständig von einer Landschaftsaufnahme eingenommen, die den Blick von einer kahlen Höhe auf Hügelketten und Täler im Nebel wiedergibt. Auf diesem Bild sind keine Menschen, Siedlungen oder Gebäude zu sehen. Der Himmel mit der tiefstehenden Sonne nimmt mehr als die Hälfte des Bildes ein. ${ }^{21}$ Mit diesen Elementen werden die Inhalte des Wanderführers eingeordnet und gedeutet. Darauf kommen wir nach der Analyse des Vorworts noch einmal zurück. Das Vorwort spricht die Nutzer:in folgendermaßen an:

Sie wollen sich auf den Weg machen in eine der schönsten Landschaften, die wir haben. Uns ist viel daran gelegen, dass Ihre Zeit hier wertvoll für Sie sein wird, denn wir wissen, wie kostbar solche Auszeiten für Sie sind. Darum geben wir Ihnen mit diesem Heft keinen gewöhnlichen Wanderführer in die Hand. Dieses Heft will Sie nicht nur auf den Wegen durch Berge und Täler begleiten; es geht auch um Höhen und Tiefen, die jedes Leben mit sich bringt.

Innere Wegbegleitung - das möchten wir Ihnen eröffnen: Ruhe, Hinschauen, Orientierung, Atem holen auch in der inneren Landschaft, die wir in uns tragen. Die meditativen Texte wollen Sie mit auf den Weg nehmen und wollen mit auf den Weg genommen werden. Im Gehen und Ruhen, im Aufbrechen und Ankommen erschließen sie sich nach und nach.

hochspirituell - kann das Erlebnis werden. Wir wünschen Ihnen den Segen dessen, der „Himmel und Erde gemacht hat“ (Psalm 124, 8)

Ihr ökumenisches Redaktionsteam

Wie in den oben analysierten Dokumenten des Klosterpfads wird die Nutzer:in des Kapellenwegführers auch hier direkt adressiert, und ihre Interessen und Bedürfnisse werden in den Vordergrund gerückt - allerdings hier nicht in Form von Wahlmöglichkeiten, sondern als Unterstellung einer Entscheidung zum Aufbruch. Als Ziel des Aufbruchs wird eine Region angenommen, die touristischer Kommunikation gemäß mit einem Superlativ ${ }^{22}$ kategorisiert wird. Sie wird zugleich einem Kollektiv (,wir“) als Besitz zugerechnet. Das „Wir“ kann zwar zunächst inklusiv angelegt sein, d.h. die Besucher:in und potenziell auch weitere Menschen sind als (Mit-)Besitzende eingeschlossen, sogar an ein gemeinsames Welterbe kann gedacht werden. Im Weiteren wird das Eigentum jedoch exklusiv auf die (touristischen) Anbieter bezogen, die den Reisenden Hilfe beim Aufenthalt versprechen. Damit wird eine asymmetrische Beziehung zwischen den Autor:innen und der Adressat:in konstituiert, in der der angesprochenen Person Zugang zum privilegiertem Wissen der (einheimischen) Autor:innen angeboten wird. Die versprochene Dienstleistung wird dabei als ganz persönliches Anliegen (,Uns ist viel daran gelegen“) eingeführt, d.h.

\footnotetext{
21 Der Kapellenwegführer hat ein Format von 21,5 cm (Höhe) und 10,5 cm (Breite); das Foto der Kruzifixe und Bilder ist $15 \mathrm{~cm}$ hoch und $5 \mathrm{~cm}$ breit.

22 Der Begriff „Landschaft“ hat eine geographische (im Sinne z. B. der Abgrenzung von „Stadt“) und eine ästhetische Dimension; sie wird betrachtet und ruft bei den Betrachtenden Empfindungen hervor. In der hohen Bewertung der Landschaft werden geteilte Kriterien unterstellt, die durch die Entscheidung der adressierten Person für dieses Reiseziel validiert wird.
} 
sie basiert auf einer intrinsisch motivierten und authentischen Selbstverpflichtung gegenüber der Nutzer:in, die nicht strategisch oder instrumentell begründet scheint. Sie wird vielmehr als individueller und unmittelbarer Dienst (,geben wir Ihnen in die Hand") gekennzeichnet. Damit setzt sich das Angebot von anderen touristischen Dienstleistungen ab. Zugleich wird aber auch an den touristischen Anlass angeschlossen, wenn auf die begrenzte Zeit des Aufenthaltes, auf seinen besonderen Wert (wertvoll, kostbar) und damit seine Außeralltäglichkeit hingewiesen wird. Der Besonderheit der „Auszeit“" entspricht das vorliegende touristische Produkt, das als nicht ,gewöhnlich“ charakterisiert wird. Da der Wanderführer durchaus den üblichen Konventionen entspricht, muss die Besonderheit in einem anderen Punkt liegen, nämlich in seiner Nutzung, die im weiteren Fortgang der Leser:in nahegelegt wird.

Der Wanderführer soll demnach als „Wegbegleitung“ verstanden werden, d.h. er soll nicht nur Orientierung bei der Wanderung gewährleisten, indem z. B. genau über die Wegeführung informiert wird, sondern er soll bei der Wanderung Gesellschaft leisten und so das Erleben teilen. Die Begleitung vollzieht sich - im Unterschied zu einem menschlichen Begleiter - nicht im Gespräch, sondern über die abgedruckten ,meditativen Texte“. Das bedeutet, die Wanderung wird nicht zusammen mit anderen Menschen unternommen (z. B. in Gruppen, wie es zum Klosterpfad angeboten wird), sondern im Zwiegespräch zwischen der wandernden Person und dem Kapellenwanderführer, das während der Bewegung im Raum nach und nach zum Verstehen der Texte führt. Diese Nutzung wird den Texten als inhärente Bestimmung und als eigene Intention (,wollen“) zugeschrieben.

Verbunden damit ist der Blick nach innen; der Betrachtung der Umgebung korrespondiert die Visualisierung des eigenen Lebens als Landschaft. ${ }^{23}$ Diese vorgeschlagene Nutzung des Kapellenführers basiert auf der Metaphorisierung von Landschaft, die den ganzen Text durchzieht: Ist am Anfang noch von einer konkreten (besonders schönen) Landschaft ${ }^{24}$ die Rede, wird dann der Lebensverlauf mit einer Wanderung verglichen und schließlich eine ,innere Wegbegleitung“ angeboten, also eine Begleitung bei der Beschäftigung mit sich selbst. Dabei wird eine Parallelität der mit der Betrachtung der inneren und der äußeren Landschaft verbundenen Prozesse unterstellt: „Ruhe, Hinschauen, Orientierung, Atem holen auch in der inneren Landschaft, die wir in uns tragen" - beide Perspektiven sind mit denselben Aktivitäten verbunden. Es sind Aktivitäten, die körperbezogen und ganzheitlich sind; sie erscheinen einerseits absichtslos, sind andererseits aber auch bewusst und gerichtet (,Hinschauen“). Sie konstituieren zusammen eine Haltung der Aufmerksamkeit und Achtsamkeit gegenüber der Landschaft und der eigenen Person. Damit wird im Text auf aktuelle Diskurse der Selbstthematisierung und der therapeutisierten Selbstbeobachtung rekurriert, die in hohem Maße anschlussfähig sind an indivi-

\footnotetext{
23 Hartmut Rosa konstatiert, dass ,die (auf den ersten Blick paradox anmutende) Idee, man müsse auf die Natur hören (oder in die Natur gehen), um sich selbst zu finden, in der Moderne eine ebenso vielfältige wie wirkmächtige und zählebige Gestalt annehmen konnte“ (2016, S. 456; Herv. i.O.). Er sieht darin ein Weiterleben der ,in ihrem Ursprung romantische(n) Idee einer heimlichen Korrespondenz oder Resonanz zwischen innerer und äußerer Natur" (ebd.).

24 Mit dem Wandern und dem Blick auf schöne Landschaften verbindet sich ein kollektives Bildgedächtnis, das in einer Ausstellung der Berliner Nationalgalerie (Verwiebe und Montura 2018) dokumentiert wurde.
} 
dualisierte, erfahrungsbezogene und ganzheitliche Formen von Religion, wie sie die moderne Spiritualität verkörpert (Knoblauch 2005). In diesem Sinne wird die „hochspirituelle“ Interpretation des Wanderns nicht vorgeschrieben, sondern bleibt der Entscheidung der Nutzer:in - ihrer Aufnahmebereitschaft - und dem Lauf der Dinge überlassen. Die Autor:innen des Textes - als „Ihr ökumenisches Redaktionsteam" offensichtlich kirchlich gebunden - scheinen in ihrer kirchlichen Verankerung jedoch keinen Gegensatz zu einer individualisierten Spiritualität zu sehen und erinnern damit indirekt an spirituelle Traditionen, die es auch innerhalb der christlichen Kirchen immer gegeben hat. Immerhin wird mit dem eingefügten Segenswunsch angedeutet, dass die Dynamik des Erlebens auch im engeren Sinne religiös gedeutet werden kann.

Insgesamt oszilliert der Text zwischen unterschiedlichen Deutungs- und damit Erlebnismöglichkeiten: Unter dem Label ,hochspirituell“ kann die Wanderung erstens als individuelle Selbstbegegnung konzipiert werden, sie kann zweitens mit einer romantischen Naturmystik verbunden oder drittens explizit als religiöses Erlebnis unternommen werden. Die zweite Variante wird unterstützt durch den an Bilder Caspar David Friedrichs erinnernden Blick auf die vernebelten und menschenleeren Berge und Täler auf dem einen Foto, während die dritte Variante auf das PsalmZitat und das Foto mit dem Sammelsurium religiöser Gegenstände verweist. Jedoch bleiben diese Bezüge zurückhaltend, denn die religiöse Zugehörigkeit schreiben sich nur die Autor:innen selbst zu, und die religiöse Botschaft des Segenswunsches wird mit distanzierenden Anführungszeichen versehen. Die Vielfalt der nebeneinander ausgestellten religiösen Devotionalien verweist einerseits darauf, dass sie Ausstattungsgegenstände der auf den Wanderwegen zu besichtigenden Kapellen sind, andererseits bringen sie die Diversität christlicher (genauer: katholischer) Religion zum Ausdruck.

\section{Resümee und Ausblick}

In diesem Beitrag sind wir am Beispiel des Wanderns dem Verhältnis von Religion und Tourismus nachgegangen. Ausgehend von differenzierungstheoretischen Überlegungen haben wir vor allem die wechselseitigen Bezugnahmen touristischer und religiöser Kommunikation ins Zentrum der Betrachtungen gerückt. An den Fallanalysen aus dem Schwarzwald wird deutlich, wie ein durch Artefakte (Gebäude, Kreuze, Wallfahrts- und Kreuzwege, Andachtsstätten usw.) religiös markierter Raum touristisch überformt und in einen touristischen Raum transformiert wird. Es ist eine Landschaft, in der Hofkapellen oder Klosterruinen als Relikte der Vergangenheit noch herumstehen, ihre Funktion aber oft verloren haben. Zugleich hat sich der Blick auf die Landschaft geändert: Sie ruft nicht mehr die Mühen und Gefahren harter landwirtschaftlicher Arbeit vor Augen, und sie ist nicht mehr durch eine Aufteilung in Besitztümer religiöser (und nicht-religiöser) Herrschaft strukturiert. Vielmehr wird sie nun kommunikativ als Ort erlebnisförmiger Erholung entworfen. Jedoch werden die religiösen Markierungen durch die touristische Aneignung konserviert und in die Aufmerksamkeit der touristischen Besucher:innen gerückt. 
Wir haben dies als touristische Recodierung von Religion sowie umgekehrt als religiöse Recodierung des Tourismus interpretiert. Typischer Ausdruck der touristischen Bezugnahmen auf Religion sind Formen der Musealisierung und Kommodifizierung des Religiösen. Religion kommt hier als kulturelles Erbe und Teil vergangener Lebenswelten ins Spiel oder bildet eine verkaufsfördernde Kulisse und Kontextualisierung für verschiedene Angebote, in denen es vor allem um Wahlmöglichkeiten und Genuss geht. Interessant ist an dieser Stelle, dass auch religiöse Akteure solche letztlich distanzierenden Reden über Religion bedienen. Diese wechselseitigen kommunikativen Anleihen und Indienstnahmen können als touristische Recodierungen verstanden werden. Neben der Politisierung, Ästhetisierung oder Verwissenschaftlichung des Religiösen kann man daher mit Blick auf unsere Befunde auch von Prozessen der Touristifizierung des Religiösen sprechen. Insofern man den Tourismus als Ergebnis einer Popularisierung des Reisens begreift, muss man davon ausgehen, dass auch diejenigen religiösen Orte und Räume einer Popularisierung unterzogen werden, die durch ihn in den Fokus gerückt werden. Da aber Religion touristisch recodiert wird - und dabei eben nicht mehr Religion bleibt -, ist dies aus der Perspektive religiöser Akteure und Institutionen ein durchaus ambivalenter Effekt. Religion wird zwar sichtbar, aber nicht als Religion, sondern als Interesse generierender Faktor für touristische Destinationen.

Als anregend für eine weitergehende Diskussion unserer Befunde könnte sich das Konzept der Dispersion des Religiösen von Michael N. Ebertz (2018) erweisen. Bei der Auflistung von Sphären, in die substantiale Religion versickert (2018, S. 417) oder in denen Religion der Dekonstruktion, Deformation oder Inversion (S. 418) unterzogen werden kann, nennt Ebertz auch den Tourismus (S. 415), ohne dass dies an dieser Stelle jedoch weiter ausgeführt wird. In weiteren Studien wäre daher die Tragfähigkeit des Dispersionskonzepts für die Untersuchung des Verhältnisses von Religion und Tourismus empirisch zu explorieren. In unseren Analysen gehen wir auch auf den Fall einer religiösen Recodierung des Tourismus ein. Hier wird die Grenze hin zu einer Form genuin religiöser Rede überschritten. Im vorliegenden Datenmaterial handelt es sich um die spirituelle Aufladung der durchwanderten Landschaft, die Anschlüsse herstellt an religiöse Themen. Hier entsteht tatsächlich auch im engeren Sinne eine religionsproduktive Kontaktzone. Mit Krech (2019) kann dies als (Re-)Sakralisierung eines touristisch konstruierten Raums gedeutet werden, mit Ebertz könnte man an dieser Stelle von „Diffusion“ oder „Synkretismus“ (2018, S. 418) sprechen. Zu vermuten ist, dass solche Bezugnahmen auch in anderen Fällen primär durch religiöse Akteure erfolgen, wobei an konfessionell gebundene oder kirchliche beauftragte Akteure ebenso zu denken ist wie an Vertreter:innen einer individuellen, konfessionell ungebundenen und möglicherweise diffusen Spiritualität.

Darüber hinaus ist eine interessante Frage, welche Funktion und welche intendierten, aber auch unintendierten Effekte diese wechselseitigen Bezugnahmen für Religion und Tourismus haben. Der von uns ins Feld geführte verkaufsfördernde Charakter religiöser Bezüge beispielsweise ist genau genommen noch eher Behauptung als ein tatsächlich nachgewiesener Effekt. Es bräuchte mehr und auch anderes Material, um einen solchen Zusammenhang wirklich rekonstruieren zu können. Aufschlussreich wäre sicherlich auch, das Verhältnis von Religion und Tourismus im 
Kontext der heritage studies (Ashley und Frank 2016; Astor et al. 2019) zu diskutieren. Da Religiöses als Teil des kulturellen Erbes verstanden werden kann, wäre in einer kultur- oder auch einer bewertungssoziologischen Perspektive zu untersuchen, auf welche Weise Religiöses zum kulturellen Erbe gemacht wird und damit auch zur kulturhistorisch interessanten touristischen Destination werden kann. Gerade Auszeichnungen als „Weltkulturerbe“ können Ansatzpunkte für touristische Kommunikation und dementsprechende Marketing-Strategien sein.

An dieser Stelle zeigt sich deutlich der Bedarf weiterer Forschungen. Die vorliegenden Befunde können hier nur eine erste Sondierung leisten und mögliche Vergleichshorizonte aufzeigen. So wäre es interessant, die um die untersuchten Wanderwege herum etablierten Kommunikationsstrategien mit solchen zu vergleichen, die sich auf traditionelle religiöse Formen des Wanderns beziehen, beispielsweise auf Pilger- und Kreuzwege oder Wallfahrtsorte. Eine weitere Vergleichsmöglichkeit bietet sich in Bezug auf eher stationäre Phänomene an, wie sie Kirchen, Heiligtümer und Friedhöfe darstellen.

Danksagung Wir danken den anonymen Gutachter:innen und Meike Haken für ihre hilfreichen Hinweise zur Überarbeitung des Manuskripts. Kerstin Menzel danken wir für ihre Ideen und Anregungen bei der gemeinsamen Dateninterpretation.

Funding Open Access funding enabled and organized by Projekt DEAL.

Open Access Dieser Artikel wird unter der Creative Commons Namensnennung 4.0 International Lizenz veröffentlicht, welche die Nutzung, Vervielfältigung, Bearbeitung, Verbreitung und Wiedergabe in jeglichem Medium und Format erlaubt, sofern Sie den/die ursprünglichen Autor(en) und die Quelle ordnungsgemäß nennen, einen Link zur Creative Commons Lizenz beifügen und angeben, ob Änderungen vorgenommen wurden.

Die in diesem Artikel enthaltenen Bilder und sonstiges Drittmaterial unterliegen ebenfalls der genannten Creative Commons Lizenz, sofern sich aus der Abbildungslegende nichts anderes ergibt. Sofern das betreffende Material nicht unter der genannten Creative Commons Lizenz steht und die betreffende Handlung nicht nach gesetzlichen Vorschriften erlaubt ist, ist für die oben aufgeführten Weiterverwendungen des Materials die Einwilligung des jeweiligen Rechteinhabers einzuholen.

Weitere Details zur Lizenz entnehmen Sie bitte der Lizenzinformation auf http://creativecommons.org/ licenses/by/4.0/deed.de.

\section{Literatur}

Andrack, Manuel. 2011. Das neue Wandern: Unterwegs auf der Suche nach dem Glück. Berlin: Verlag Taschenbuch.

Ashley, Susan L.T., und Sybille Frank. 2016. Introduction: heritage-outside-in. International Journal of Heritage Studies 22(7):501-503. https://doi.org/10.1080/13527258.2016.1184704.

Astor, Avi, Marian Burchardt, und Mar Griera. 2019. Polarization and the limits of politicization: Cordoba's Mosque-Cathedral and the politics of cultural heritage. Qualitative Sociology 42:337-360.

Bourdieu, Pierre. 1999. Die Regeln der Kunst. Genese und Struktur des literarischen Feldes. Frankfurt a.M.: Suhrkamp.

Ebertz, Michael N. 2018. Dispersion und Transformation. In Handbuch Religionssoziologie, Hrsg. Detlef Pollack, Volkhard Krech, Olaf Müller und Markus Hero, 411-436. Wiesbaden: Springer VS.

Elias, Norbert. 1983. Der Fußballsport im Prozess der Zivilisation. In Der Satz „Der Ball ist rund“ hat eine gewisse philosophische Tiefe, Hrsg. Rolf Lindner, 12-21. Berlin: Transit.

Enzensberger, Hans Magnus. 1979. Eine Theorie des Tourismus. In Einzelheiten I-Bewußtseins-Industrie, 179-205. Frankfurt a.M.: Suhrkamp. (1958/1979). 
Gerhardts, Jürgen. 2001. Der Aufstand des Publikums. Eine systemtheoretische Interpretation des Kulturwandels in Deutschland zwischen 1960 und 1989. Zeitschrift für Soziologie 30(3):163-184.

Graefe, Lena. 2021. Der deutsche Reisemarkt - Zahlen und Fakten 2020. Statista. Erhebung durch die Gesellschaft für Konsumforschung (GfK). Veröffentlicht durch den Deutschen Reiseverband (DRV). https://de.statista.com/statistik/daten/studie/182432/umfrage/gesamtumsatz-der-deutschenreiseveranstalter-seit-2004/. Zugegriffen: 15. Febr. 2021.

Hachtmann, Rüdiger. 2010. Tourismus und Tourismusgeschichte, Version: 1.0. Docupedia-Zeitgeschichte, 22.12.2010. http://docupedia.de/zg/hachtmann_tourismusgeschichte_v1_de_2010. Zugegriffen: 21. Febr. 2021. https://doi.org/10.14765/zzf.dok.2.312.v1.

Hennig, Christoph. 1999. Reiselust. Touristen, Tourismus und Urlaubskultur. Frankfurt a.M.: Suhrkamp.

Karstein, Uta. 2021. Geschmackserziehung im „Kitschzeitalter“. Zur Formierung der Sinne im 19. Jahrhundert. In: Wahrnehmung als soziale Praxis, Hrsg. Christiane Schürkmann, Nina Tessa Zahner, 111-132. Wiesbaden, Springer VS

Karstein, Uta; Zahner, Nina. 2015. Anfragen an die Soziologie des Publikums. In: Routinen der Krise Krise der Routinen. Verhandlungen des 37. Kongresses der Deutschen Gesellschaft für Soziologie in Trier 2014, Hrsg. Stephan Lessenich (CD-Rom), Wiesbaden, Springer VS

Kerkeling, Hape. 2006. Ich bin dann mal weg: Meine Reise auf dem Jakobsweg. München: Piper.

Knoblauch, Hubert. 2005. Einleitung: Soziologie der Spiritualität. Zeitschrift für Religionswissenschaft 13(2):123-132.

Knoblauch, Hubert. 2009. Populäre Religion: Auf dem Weg in eine spirituelle Gesellschaft. Frankfurt a.M.: Campus.

Krech, Volkhard. 2011. Wo bleibt die Religion? Zur Ambivalenz des Religiösen in der modernen Gesellschaft. Bielefeld: transcript.

Krech, Volkhard. 2019. Operative Durchlässigkeit oder Schließung der Religion? Eine differenzierungstheoretische Lesart der Religionssoziologie von Wolfgang Eßbach. Zeitschrift für Religion, Gesellschaft und Politik 3(1):103-130.

Kretschmann, Carsten (Hrsg.). 2003. Wissenspopularisierung: Konzepte der Wissensverbreitung im Wandel. Wissenskultur und gesellschaftlicher Wandel. Berlin: de Gruyter.

Löw, Martina. 2001. Raumsoziologie. Frankfurt a.M.: Suhrkamp

Luhmann, Niklas. 1987a. Läßt unsere Gesellschaft Kommunikation mit Gott zu? In Soziologische Aufklärung 4. Beiträge zur funktionalen Differenzierung der Gesellschaft, 227-235. Opladen: Westdeutscher Verlag.

Luhmann, Niklas. 1987b. Die Unterscheidung Gottes. In Soziologische Aufklärung 4. Beiträge zur funktionalen Differenzierung der Gesellschaft, 236-253. Opladen: Westdeutscher Verlag.

Luhmann, Niklas. 1998. Religion als Kommunikation. In Religion als Kommunikation, Hrsg. Hartmann Tyrell, Volkhard Krech, und Hubert Knoblauch, 135-145. Würzburg: Ergon.

Makropolus, Michael. 2008. Theorie der Massenkultur. München: Wilhelm Fink.

Norris, Pippa, und Ronald Inglehart. 2004. Sacred and secular. Religion and politics worldwide. Cambridge, New York: Cambridge University Press.

Oevermann, Ulrich. 1983. Zur Sache. Die Bedeutung von Adornos methodologischem Selbstverständnis für die Begründung einer materialen soziologischen Strukturanalyse. In Adorno-Konferenz 1983, Hrsg. Ludwig von Friedeburg, Jürgen Habermas, 234-289. Frankfurt a.M.: Suhrkamp.

Oevermann, Ulrich. 2000. Die Methode der Fallrekonstruktion in der Grundlagenforschung sowie der klinischen und pädagogischen Praxis. In Die Fallrekonstruktion. Sinnverstehen in der sozialwissenschaftlichen Forschung, Hrsg. Klaus Kraimer, 58-156. Frankfurt a.M.: Suhrkamp.

Palatschek, Silvia (Hrsg.). 2008. Popularisierung von Wissenschaft. Sonderheft der Zeitschrift Historische Anthropologie, Bd. 16(1). Köln, Weimar, Wien: Böhlau Verlag.

Pechlaner, Harald, und Michael Volgger. 2017. Einleitung: Die Gesellschaft auf Reisen - Reise in die Gesellschaft. In Die Gesellschaft auf Reisen - Reise in die Gesellschaft, Hrsg. Harald Pechlaner, Michael Volgger, 1-10. Wiesbaden: Springer VS.

Pollack, Detlef. 1995. Was ist Religion? Probleme der Definition. Zeitschrift für Religionswissenschaft 3:163-190.

Pott, Andreas. 2011. Die Raumordnung des Tourismus. Soziale Systeme 17(2):255-276.

Rosa, Hartmut. 2016. Resonanz. Eine Soziologie der Weltbeziehung. Berlin: Suhrkamp.

Sammet, Kornelia. 2006. Die Wahrnehmung des Islam als Religion und als Kultur. Kommunikative Muster in den Gruppendiskussionen zur Frage des Moscheebaus. In Kirche in der Vielfalt der Lebensbezüge. Die vierte EKD-Erhebung über Kirchenmitgliedschaft. Band 2: Analysen zu Gruppendiskussionen und Erzählinterviews. Hrsg. Jan Hermelink, Ingrid Lukatis und Monika Wohlrab-Sahr, 293-317. Gütersloh: Gütersloher Verlag. 
Sammet, Kornelia und Franz Erhard. 2018. Methodologische Grundlagen und praktische Verfahren der Sequenzanalyse. Eine didaktische Einführung. In Sequenzanalyse Praktisch. Hrsg. Franz Erhard und Kornelia Sammet, 15-72. Weinheim: Beltz Juventa.

Schäfer, Robert. 2015. Tourismus und Authentizität. Zur gesellschaftlichen Organisation von Außeralltäglichkeit. Bielefeld: transcript.

Scheuch, Erwin K. 1969. Soziologie der Freizeit. In Handbuch der Empirischen Sozialforschung, Hrsg. René König, 735-833. Stuttgart: Ferdinand Enke Verlag.

Sieger, Marvin, und Christoph Bendzulla. 2018. Mittels Schilde im Bilde. Zur Erzeugung eines Naturschutzgebietes im Medium des Schildes. In Sequenzanalyse praktisch, Hrsg. Franz Erhard, Kornelia Sammet, 244-270. Weinheim, Basel: Beltz Juventa.

Simmel, Georg. 1992. Soziologie. Untersuchungen über die Formen der Vergesellschaftung. Frankfurt a.M.: Suhrkamp.

Sloterdijk, Peter. 2017. Tractatus philosophico-touristico. In Die Gesellschaft auf Reisen - eine Reise in die Gesellschaft, Hrsg. Harald Pechlaner, Michael Volgger, 11-22. Wiesbaden: Springer VS.

Stausberg, Michael. 2010. Religion im modernen Tourismus. Berlin: Suhrkamp.

Stichweh, Rudolf. 1988. Inklusion in Funktionssysteme der modernen Gesellschaft. In Differenzierung und Verselbständigung. Zur Entwicklung gesellschaftlicher Teilsysteme, Hrsg. Renate Mayntz, et al., 261-294. Frankfurt a.M.: Campus.

Stichweh, Rudolf. 1994. Wissenschaft, Universität, Professionen. Frankfurt a.M.: Suhrkamp.

Strauss, Anselm. 1991. Grundlagen qualitativer Sozialforschung. Datenanalyse und Theoriebildung in der empirischen soziologischen Forschung. München: Fink. (1987).

Strauss, Anselm, und Juliet Corbin. 1996. Grounded Theory. Grundlagen qualitativer Sozialforschung. Weinheim: Psychologie-Verl.-Union.

Verwiebe, Birgit, und Gabriel Montura. 2018. Wanderlust, Von Caspar David Friedrich bis Auguste Renoir. München: Hirmer.

Weber, Max. 1980. Wirtschaft und Gesellschaft. Grundriß der verstehenden Soziologie, 5. Aufl., besorgt von Johannes Winckelmann. Tübingen: Mohr. 